OHSTPY-HEP-T-99-022

$\mathrm{MZ}-\mathrm{TH} / 99-64$

\title{
One-Loop Self Energy and Renormalization of the Speed of Light for some Anisotropic Improved Quark Actions
}

\author{
Stefan Groote \\ Newman Laboratory, Cornell University, Ithaca, NY 14853, USA \\ and \\ Institut für Physik der Johannes-Gutenberg-Universität, \\ Staudingerweg 7, D-55099 Mainz, Germany \\ Junko Shigemitsu \\ Physics Department, The Ohio State University, Columbus, OH 43210, USA
}

\begin{abstract}
One-loop corrections to the fermion rest mass $M_{1}$, wave function renormalization $Z_{2}$ and speed of light renormalization $C_{0}$ are presented for lattice actions that combine improved glue with clover or D234 quark actions and keep the temporal and spatial lattice spacings, $a_{t}$ and $a_{s}$, distinct. We explore a range of values for the anisotropy parameter $\chi \equiv a_{s} / a_{t}$ and treat both massive and massless fermions.
\end{abstract}

PACS number(s): 11.10.Gh, 11.15.Ha, 12.38.Bx, 12.38.Gc

Typeset using REVTEX 


\section{INTRODUCTION}

Examples of successful employment of anisotropic lattices in lattice QCD simulations have been increasing lately. They include extensive studies of the glueball spectrum [1], investigations of heavy hybrid states [2,3] and calculations of quarkonium fine structure [4]. In most cases one is dealing with large states requiring large spatial volumes and also signals that can only be extracted from high statistics data. Working with highly improved actions on coarse lattices helps with the large volume and statistics problems, however, a coarse temporal lattice spacing means that correlation functions fall off very rapidly. This last problem can be circumvented by going to an anisotropic lattice which allows for a much finer temporal grid. The correlation functions can be sampled much more frequently in a given physical time region where the signal is still good.

Another potential use of anisotropic lattices would be in simulations of matrix elements in hadronic states with large momenta. These typically occur in semileptonic decays of heavy hadrons. Once one goes beyond spectrum calculations to matrix elements, one is faced with the matching problem between operators in the coarse highly improved lattice theory and continuum QCD. In this article we take a first step in accumulating necessary renormalization factors based on perturbation theory. We carry out the one-loop self energy calculation in several anisotropic improved quark actions. This gives us renormalization of the rest mass $M_{1}$, the wave function renormalization $Z_{2}$ and the "speed of light" renormalization, $C_{0}$, a quantity which will be defined more precisely in the following sections. We treat both massive and massless quarks. Perturbation theory can be used not only in operator matchings, but also to fix parameters in the lattice actions. $C_{0}$ is an example of one such parameter.

In the next section we introduce the gauge and quark actions considered in this article. Section 3 describes the general formalism that we employ for the self energy calculation with massive fermions. We follow closely the work of the Fermilab group [5] which we could straightforwardly extend to anisotropic actions. Section 4 discusses specific one-loop contributions for mass, wavefunction and speed of light renormalizations. Our results are tabulated in section 5 for various choices of actions, fermion masses and degree of anisotropy. Some calculational details are left for appendices, where we describe Feynman rules and IR subtractions in our calculations.

\section{GAUGE AND QUARK ACTIONS}

We work with two classes of gauge actions denoted $\mathcal{S}_{G}^{I}$ and $\mathcal{S}_{G}^{I I}$ [6,7], with

$$
\begin{aligned}
\mathcal{S}_{G}^{I}= & -\beta \sum_{x, s>s^{\prime}} \frac{1}{\chi}\left\{c_{0}^{G} \frac{P_{s s^{\prime}}}{u_{s}^{4}}+c_{1}^{G} \frac{R_{s s^{\prime}}}{u_{s}^{6}}+c_{1}^{G} \frac{R_{s^{\prime} s}}{u_{s}^{6}}\right\} \\
& -\beta \sum_{x, s} \chi\left\{c_{0}^{G} \frac{P_{s t}}{u_{s}^{2} u_{t}^{2}}+c_{1}^{G} \frac{R_{s t}}{u_{s}^{4} u_{t}^{2}}+c_{1}^{G} \frac{R_{t s}}{u_{t}^{4} u_{s}^{2}}\right\}
\end{aligned}
$$

and 


$$
\begin{aligned}
\mathcal{S}_{G}^{I I}= & -\beta \sum_{x, s>s^{\prime}} \frac{1}{\chi}\left\{\frac{5}{3} \frac{P_{s s^{\prime}}}{u_{s}^{4}}-\frac{1}{12} \frac{R_{s s^{\prime}}}{u_{s}^{6}}-\frac{1}{12} \frac{R_{s^{\prime} s}}{u_{s}^{6}}\right\} \\
& -\beta \sum_{x, s} \chi\left\{\frac{4}{3} \frac{P_{s t}}{u_{s}^{2} u_{t}^{2}}-\frac{1}{12} \frac{R_{s t}}{u_{s}^{4} u_{t}^{2}}\right\} .
\end{aligned}
$$

The $x$ sum is over lattice sites and the variable $s$ runs over spatial directions. $\beta \equiv 2 N_{c} / g^{2}$, $\chi$ is the anisotropy parameter

$$
\chi=a_{s} / a_{t}
$$

and

$$
\begin{aligned}
& P_{\mu \nu}=\frac{1}{N_{c}} \operatorname{Real}\left(\operatorname{Tr}\left\{U_{\mu}(x) U_{\nu}\left(x+a_{\mu}\right) U_{\mu}^{\dagger}\left(x+a_{\nu}\right) U_{\nu}^{\dagger}(x)\right\}\right), \\
& R_{\mu \nu}=\frac{1}{N_{c}} \operatorname{Real}\left(\operatorname{Tr}\left\{U_{\mu}(x) U_{\mu}\left(x+a_{\mu}\right) U_{\nu}\left(x+2 a_{\mu}\right) U_{\mu}^{\dagger}\left(x+a_{\mu}+a_{\nu}\right) U_{\mu}^{\dagger}\left(x+a_{\nu}\right) U_{\nu}^{\dagger}(x)\right\}\right) .
\end{aligned}
$$

$u_{s}$ and $u_{t}$ are the tadpole improvement parameters $u_{0}$ for spatial and temporal link variables respectively [8].

The parameters $c_{0}^{G}$ and $c_{1}^{G}$ in action $\mathcal{S}_{G}^{I}$ are constrained to satisfy $c_{0}^{G}+8 c_{1}^{G}=1$. The Symanzik improved gauge action, in which $O\left(a^{2}\right)$ errors are removed, corresponds to $c_{0}^{G}=$ $5 / 3$ and $c_{1}^{G}=-1 / 12$ [9], whereas $c_{0}^{G}=3.648$ and $c_{1}^{G}=-0.331$, for $\chi=1$, leads to one of the RG improved Iwasaki actions [10]. In $\mathcal{S}_{G}^{I I}$ parameters have been fixed to the Symanzik values. We will be working mainly with Symanzik improved actions and present RG improved results only for a few cases. We note that the action $\mathcal{S}_{G}^{I I}$ is intrinsically asymmetric even for the isotropic limit $\chi=1$.

The most highly improved quark action that we have analysed is the D234 action [6].

$$
\begin{aligned}
\mathcal{S}_{D 234}^{I}= & a_{s}^{3} a_{t} \sum_{x} \bar{\Psi}_{c}\left\{\gamma_{t} \frac{1}{a_{t}}\left(\nabla_{t}-\frac{1}{6} C_{3 t} \nabla_{t}^{(3)}\right)+\frac{C_{0}}{a_{s}} \vec{\gamma} \cdot\left(\vec{\nabla}-\frac{1}{6} C_{3} \vec{\nabla}^{(3)}\right)+m_{0}\right. \\
& -\frac{r a_{s}}{2}\left[\frac{1}{a_{t}^{2}}\left(\nabla_{t}^{(2)}-\frac{1}{12} C_{4 t} \nabla_{t}^{(4)}\right)+\frac{1}{a_{s}^{2}} \sum_{j}\left(\nabla_{j}^{(2)}-\frac{1}{12} C_{4} \nabla_{j}^{(4)}\right)\right] \\
& \left.-r a_{s} \frac{C_{F}}{4} \frac{i \sigma_{\mu \nu} \tilde{F}^{\mu \nu}}{a_{\mu} a_{\nu}}\right\} \Psi_{c} \\
= & \sum_{x} \bar{\Psi}_{L}\left\{\gamma_{t}\left(\nabla_{t}-\frac{1}{6} C_{3 t} \nabla_{t}^{(3)}\right)+\frac{C_{0}}{\chi} \vec{\gamma} \cdot\left(\vec{\nabla}-\frac{1}{6} C_{3} \vec{\nabla}^{(3)}\right)+a_{t} m_{0}\right. \\
& -\frac{r}{2}\left[\chi\left(\nabla_{t}^{(2)}-\frac{1}{12} C_{4 t} \nabla_{t}^{(4)}\right)+\frac{1}{\chi} \sum_{j}\left(\nabla_{j}^{(2)}-\frac{1}{12} C_{4} \nabla_{j}^{(4)}\right)\right] \\
& \left.-r \frac{C_{F}}{4} i \sigma_{\mu \nu} \tilde{F}^{\mu \nu} \frac{a_{s} a_{t}}{a_{\mu} a_{\nu}}\right\} \Psi_{L} .
\end{aligned}
$$

The quark fields $\Psi_{c}$ and the dimensionless lattice fields $\Psi_{L}$ are related through

$$
\Psi_{L}=a_{s}^{3 / 2} \Psi_{c}
$$


The dimensionless derivatives $\nabla^{(n)}$ and field strength tensors $\tilde{F}^{\mu \nu}$ are tadpole improved [8] and defined in the Appendix. We use the convention $\sigma_{\mu \nu}=\frac{1}{2}\left[\gamma_{\mu}, \gamma_{\nu}\right]$ and set $r=1$ in all our calculations. At tree-level the coefficients $C_{0}, C_{3}, C_{3 t}, C_{4}, C_{4 t}$ and $C_{F}$ are equal to one. The quark action is then tree-level accurate through $O\left(a_{s}^{3}\right)$ and $O\left(a_{t}^{3}\right) . C_{0}$ is what we call the "speed of light". This parameter is adjusted, in general either perturbatively or nonperturbatively, to ensure correct dispersion relations for particles. In anticipation of working on anisotropic lattices with $a_{t}$ much finer than $a_{s}$, one can drop the higher order improvement terms in the temporal derivatives by setting $C_{3 t}=C_{4 t}=0$, without loosing accuracy. We call this action $\mathcal{S}_{D 234}^{I I}$.

$$
\begin{aligned}
\mathcal{S}_{D 234}^{I I}= & \sum_{x} \bar{\Psi}_{L}\left\{\gamma_{t} \nabla_{t}+\frac{C_{0}}{\chi} \vec{\gamma} \cdot\left(\vec{\nabla}-\frac{1}{6} C_{3} \vec{\nabla}^{(3)}\right)+a_{t} m_{0}\right. \\
& -\frac{r}{2}\left[\chi \nabla_{t}^{(2)}+\frac{1}{\chi} \sum_{j}\left(\nabla_{j}^{(2)}-\frac{1}{12} C_{4} \nabla_{j}^{(4)}\right)\right] \\
& \left.-r \frac{C_{F}}{4} i \sigma_{\mu \nu} \tilde{F}^{\mu \nu} \frac{a_{s} a_{t}}{a_{\mu} a_{\nu}}\right\} \Psi_{L}
\end{aligned}
$$

The familiar $O(a)$ accurate clover quark [11] action corresponds to setting $C_{3}=C_{4}=0$ in the above and using a less improved field strength tensor $F^{\mu \nu}$ (also defined in the Appendix) rather than $\tilde{F}^{\mu \nu}$.

$$
\begin{aligned}
\mathcal{S}_{\text {clover }}= & \sum_{x} \bar{\Psi}_{L}\left\{\gamma_{t} \nabla_{t}+\frac{C_{0}}{\chi} \vec{\gamma} \cdot \vec{\nabla}+a_{t} m_{0}\right. \\
& \left.-\frac{r}{2}\left[\chi \nabla_{t}^{(2)}+\frac{1}{\chi} \sum_{j} \nabla_{j}^{(2)}\right]-r \frac{C_{F}}{4} i \sigma_{\mu \nu} F^{\mu \nu} \frac{a_{s} a_{t}}{a_{\mu} a_{\nu}}\right\} \Psi_{L}
\end{aligned}
$$

We have carried out one-loop self energy calculations for several combinations of the above gauge and quark actions, for both massless and massive quarks. We list the specific actions considered in Table I. For actions $\mathcal{S}^{A}$ and $\mathcal{S}^{A^{\prime}}$ massless results have already appeared in [12]. We agree with their results and we include these cases here for completeness. With action $\mathcal{S}^{C}$ we treat only the massless case, since our formalism for massive quarks, following [5], requires that the only time derivatives be in the $\nabla_{t}$ and $\nabla_{t}^{(2)}$ terms. Both $\mathcal{S}_{D 234}^{I I}$ and $\mathcal{S}_{\text {clover }}$ satisfy this condition, but $\mathcal{S}_{D 234}^{I}$ does not.

\section{GENERAL FORMALISM FOR SELF ENERGY CALCULATIONS}

In this section we summarize the formalism for self energy calculations, along the lines of reference [5]. Perturbative calculations for massive Wilson quarks are also described in reference [13]. We concentrate on the massive case, since massless lattice perturbation theory has been in the literature for decades.

For massive fermions we use either $\mathcal{S}_{D 234}^{I I}$ or $\mathcal{S}_{\text {clover. }}$. The fermion self energy $\Sigma(p)$ is defined in terms of the momentum space propagators $\bar{G}(p)$ and $\bar{G}_{0}(p)$ for the full and free theories respectively, as 


$$
\bar{G}^{-1}(p)=\bar{G}_{0}^{-1}(p)-\Sigma(p)
$$

Carrying out the Fourier transform in $p_{0}$ one defines

$$
\begin{aligned}
G(t, \vec{p}) & =\int_{-\pi / a_{t}}^{\pi / a_{t}} \frac{d p_{0}}{2 \pi} e^{i p_{0} t} \bar{G}\left(p_{0}, \vec{p}\right) \\
& \equiv \mathcal{Z}_{2}(\vec{p}) e^{-E(\vec{p}) t} \Gamma_{\text {proj }}+\ldots
\end{aligned}
$$

$\Gamma_{\text {proj }}$ is a projection operator in Dirac space. The ellipses refer to lattice artifacts and additional multi-particle states that could be created by the lattice fermion field operator $\Psi$ beyond the single quark state. The rest mass, $M_{1}$, is defined as

$$
M_{1}=E(\vec{p}=\overrightarrow{0})
$$

We do not consider the kinetic mass, $M_{2}$ [5] in this article. We will renormalize at the point $\left(p_{0}, \vec{p}\right)=\left(i M_{1}, \overrightarrow{0}\right)$ and define the wave function renormalization constant

$$
Z_{2}=\mathcal{Z}_{2}(\vec{p}=\overrightarrow{0})
$$

For a zero spatial momentum quark propagating forward in time one expects $(t>0)$

$$
\begin{aligned}
G(t, 0) & =\int_{-\pi / a_{t}}^{\pi / a_{t}} \frac{d p_{0}}{2 \pi} e^{i p_{0} t} \bar{G}\left(p_{0}, 0\right) \\
& \equiv Z_{2} e^{-M_{1} t} \frac{1+\gamma_{0}}{2}+\ldots
\end{aligned}
$$

Our goal in this section is to relate $Z_{2}$ and $M_{1}$ to parameters in the action and to $\Sigma(p)$. In order to orient ourselves, however, it is useful to first consider the free case with $\Sigma(p)=0$.

\section{A. Free Anisotropic Propagator}

The free propagator $\bar{G}_{0}\left(p_{0}, \vec{p}=0\right)$ for both actions $\mathcal{S}_{D 234}^{I I}$ and $\mathcal{S}_{\text {clover }}$ becomes (for $\left.r=1\right)$

$$
\begin{aligned}
\frac{1}{a_{t}} \bar{G}_{0}\left(p_{0}, \vec{p}=0\right) & =\frac{1}{i \gamma_{0} \sin \left(a_{t} p_{0}\right)+a_{t} m_{0}+\chi-\chi \cos \left(a_{t} p_{0}\right)} \\
& =\frac{-i \gamma_{0} \sin \left(a_{t} p_{0}\right)+a_{t} m_{0}+\chi-\chi \cos \left(a_{t} p_{0}\right)}{\left(\sin \left(a_{t} p_{0}\right)\right)^{2}+\left[\left(a_{t} m_{0}+\chi\right)-\chi \cos \left(a_{t} p_{0}\right)\right]^{2}} .
\end{aligned}
$$

In terms of the variable

$$
z \equiv e^{i a_{t} p_{0}}=e^{-a_{t} E}
$$

$\left(p_{0}=i E\right)$, one finds two zeros of the denominator corresponding to positive energy solutions.

$$
z_{1}=\frac{\left(a_{t} m_{0}+\chi\right)-\sqrt{\left(a_{t} m_{0}+\chi\right)^{2}+1-\chi^{2}}}{\chi-1}
$$


and

$$
\tilde{z}_{1}=\frac{\left(a_{t} m_{0}+\chi\right)-\sqrt{\left(a_{t} m_{0}+\chi\right)^{2}+1-\chi^{2}}}{\chi+1} .
$$

The other two zeros, $z_{2}$ and $\tilde{z}_{2}$ correspond to negative energy solutions, $z_{2}=1 / z_{1}$ and $\tilde{z}_{2}=1 / \tilde{z}_{1}$. The integral over $p_{0}$ in (15) can be done as a contour integral around the unit circle in the variable $z$. One picks up contributions from both positive energy solutions (for $t>0)$.

$$
\begin{aligned}
& \text { pole at } z_{1}: \quad \frac{\left(1+\gamma_{0}\right)}{2} \frac{e^{-M_{1}^{(0)} t}}{\sqrt{\left(a_{t} m_{0}+\chi\right)^{2}+1-\chi^{2}}}, \\
& \text { pole at } \tilde{z}_{1}: \quad \frac{\left(1-\gamma_{0}\right)}{2} \frac{e^{-\tilde{M}_{1}^{(0)} t}}{\sqrt{\left(a_{t} m_{0}+\chi\right)^{2}+1-\chi^{2}}},
\end{aligned}
$$

with

$$
a_{t} M_{1}^{(0)}=-\ln \left(z_{1}\right), \quad \quad a_{t} \tilde{M}_{1}^{(0)}=-\ln \left(\tilde{z}_{1}\right) .
$$

Clearly, $z_{1}$ is the physical positive energy solution. The second solution $\tilde{z}_{1}$ is a lattice artifact, similar to the time doubler for $r \neq 1$ in isotropic actions. The solution $\tilde{z}_{1}$ disappears in the isotropic limit, $\chi \rightarrow 1$, where $a_{t} \tilde{M}_{1}^{(0)} \rightarrow \infty$. In the same limit the physical solution $z_{1}$ goes over into the well known result

$$
z_{1} \rightarrow \frac{1}{1+a_{t} m_{0}}
$$

The gap between $\tilde{M}_{1}^{(0)}$ and $M_{1}^{(0)}$, measured in units of $1 / a_{s}$ is

$$
a_{s}\left(\tilde{M}_{1}^{(0)}-M_{1}^{(0)}\right)=\chi \ln \frac{(\chi+1)}{(\chi-1)},
$$

independent of $m_{0}$. This becomes $\infty$ at $\chi=1$ and approaches 2 as $\chi \rightarrow \infty$. The size of this gap, $a_{s} \delta E \geq 2$, is hence equal to or larger than the amount by which conventional spatial doublers are raised through the Wilson mechanism. We will henceforth ignore $\tilde{z}_{1}$ and concentrate on the physical pole at $z=z_{1}$. Comparing (20) with (15) one sees that there is nontrivial mass dependent wave function renormalization even at tree-level with

$$
Z_{2}^{(0)}=\frac{1}{\sqrt{\left(a_{t} m_{0}+\chi\right)^{2}+1-\chi^{2}}}=\frac{1}{\chi \sinh \left(a_{t} M_{1}^{(0)}\right)+\cosh \left(a_{t} M_{1}^{(0)}\right)} .
$$

This has been pointed out several times in the literature [14], [15].

A useful way to rewrite (18) is

$$
a_{t} m_{0}+\chi=\chi \cosh \left(a_{t} M_{1}^{(0)}\right)+\sinh \left(a_{t} M_{1}^{(0)}\right) .
$$




\section{B. Mass Renormalization}

In the interacting case one has a nontrivial $\Sigma(p)$ which we write as

$$
a_{t} \Sigma(p)=i \gamma_{0} B_{0}\left(p, m_{0}\right) \sin \left(a_{t} p_{0}\right)+i \frac{1}{\chi} \sum_{j}\left[\gamma_{j} B_{j}\left(p, m_{0}\right) \sin \left(a_{s} p_{j}\right)\right]+C\left(p, m_{0}\right)
$$

The $\vec{p}=0$ propagator becomes

$$
\frac{1}{a_{t}} \bar{G}\left(p_{0}, \vec{p}=0\right)=\frac{-i \gamma_{0}\left(1-B_{0}\right) \sin \left(a_{t} p_{0}\right)+a_{t} m_{0}+\chi-C-\chi \cos \left(a_{t} p_{0}\right)}{\left(1-B_{0}\right)^{2}\left[\sin \left(a_{t} p_{0}\right)\right]^{2}+\left[\left(a_{t} m_{0}+\chi\right)-C-\chi \cos \left(a_{t} p_{0}\right)\right]^{2}},
$$

where, $B_{0}=B_{0}\left(p_{0}, m_{0}\right)$ and $C=C\left(p_{0}, m_{0}\right)$ are evaluated at $\vec{p}=0$. If $p_{0}=i E$ is the location of a pole in (28), the following implicit equation must be satisfied.

$$
\left(1-B_{0}\left(i E, m_{0}\right)\right) \sinh \left(a_{t} E\right)= \pm\left[\left(a_{t} m_{0}+\chi\right)-C\left(i E, m_{0}\right)-\chi \cosh \left(a_{t} E\right)\right]
$$

One can check that the "+" sign leads to the pole $M_{1}^{(0)}$ in the free limit. Hence, the implicit equation for $M_{1}$ is given by

$$
\chi \cosh \left(a_{t} M_{1}\right)+\sinh \left(a_{t} M_{1}\right)=a_{t} m_{0}+\chi+B_{0}\left(i M_{1}, m_{0}\right) \sinh \left(a_{t} M_{1}\right)-C\left(i M_{1}, m_{0}\right) .
$$

In a perturbative calculation of $M_{1}$ one expands

$$
M_{1}=M_{1}^{(0)}+\alpha_{s} M_{1}^{(1)}+O\left(\alpha_{s}^{2}\right) .
$$

$B_{0}$ and $C$ in (30) start out $O\left(\alpha_{s}\right)$, so through one-loop their argument can be replaced by the tree-level $M_{1}^{(0)}$. Expanding the LHS also through $O\left(\alpha_{s}\right)$ and taking (26) into account, one finds

$$
\begin{aligned}
\alpha_{s} a_{t} M_{1}^{(1)} & =\frac{B_{0}\left(i M_{1}^{(0)}, m_{0}\right) \sinh \left(a_{t} M_{1}^{(0)}\right)-C\left(i M_{1}^{(0)}, m_{0}\right)}{\chi \sinh \left(a_{t} M_{1}^{(0)}\right)+\cosh \left(a_{t} M_{1}^{(0)}\right)} \\
& =-Z_{2}^{(0)} \operatorname{tr}\left\{\frac{\left(\gamma_{0}+1\right)}{4} a_{t} \Sigma\left(p_{0}=i M_{1}^{(0)}, \vec{p}=0\right)\right\},
\end{aligned}
$$

where the trace is taken over Dirac space. We note that in the $M_{1}^{(0)}=0, m_{0}=0$ limit, the $\gamma_{0}$ part of the trace $\operatorname{tr}\left\{\left(\gamma_{0}+1\right) \Sigma\right\}$ does not contribute and one has

$$
\alpha_{s} a_{t} M_{1}^{(1)}(0)=-\operatorname{tr}\left\{a_{t} \Sigma(0)\right\} / 4=-C(0,0) .
$$

In order to have massless quarks remain massless under renormalization, one needs to carry out additive mass renormalization and $M_{1}^{(1)}$ in (32) requires a subtraction. This subtraction

must be done without jeopardizing the pole condition (30). There is a standard way to accomplish this. Let $m_{c}$ be the value of the bare quark mass parameter $m_{0}$ for which the physical quark rest mass vanishes $\left(M_{1}=0\right)$. Eqn.(30) then tells us that $m_{c}$ is implicitly defined through 


$$
a_{t} m_{c}-C\left(0, m_{c}\right)=0 .
$$

In equations such as (28) or (30) one always has the combination $a_{t} m_{0}-C$. Using (34) one can add and subtract $a_{t} m_{c}$ so that

$$
a_{t} m_{0}-C \rightarrow a_{t}\left(m_{0}-m_{c}\right)-\left(C-C\left(0, m_{c}\right)\right)=a_{t} m-\tilde{C} .
$$

Previous derivations go through with $m_{0}$ replaced by

$$
m \equiv m_{0}-m_{c}
$$

and $C\left(i M_{1}, m_{0}\right)$ by

$$
\tilde{C}\left(i M_{1}, m_{0}\right)=C\left(i M_{1}, m_{0}\right)-C\left(0, m_{c}\right) .
$$

In most lattice simulations, $m_{c}$ and hence also $m$ are determined nonperturbatively from the simulations themselves. For $\tilde{C}$, however, one still often uses the one-loop result

$$
\tilde{C}\left(i M_{1}^{(0)}, m_{0}\right)=\tilde{C}\left(i M_{1}^{(0)}, m\right)=C\left(i M_{1}^{(0)}, m\right)-C(0,0) .
$$

$M_{1}^{(0)}$ is now given in terms of $m$ rather than $m_{0}$. We will be presenting our results as functions of $a_{s} M_{1}^{(0)}$, with the understanding that the shift $m_{0} \rightarrow m_{0}-m_{c}$ has been carried out and that, for instance, $M_{1}^{(0)}$ is given by

$$
a_{t} m+\chi=\chi \cosh \left(a_{t} M_{1}^{(0)}\right)+\sinh \left(a_{t} M_{1}^{(0)}\right)
$$

rather than by (26). In (32) one needs to replace $C$ by $\tilde{C}$. Our final formula for the one-loop mass correction, measured in units of $1 / a_{s}$ then becomes

$$
\begin{aligned}
\alpha_{s} a_{s} M_{1, \text { sub }}^{(1)} & =\chi \frac{B_{0}\left(i M_{1}^{(0)}, m\right) \sinh \left(a_{t} M_{1}^{(0)}\right)-\tilde{C}\left(i M_{1}^{(0)}, m\right)}{\chi \sinh \left(a_{t} M_{1}^{(0)}\right)+\cosh \left(a_{t} M_{1}^{(0)}\right)} \\
& =-Z_{2}^{(0)} \operatorname{tr}\left\{\frac{\left(\gamma_{0}+1\right)}{4}\left[a_{s} \Sigma\left(p_{0}=i M_{1}^{(0)}, \vec{p}=0, m\right)-a_{s} \Sigma(0, \overrightarrow{0}, 0)\right]\right\} .
\end{aligned}
$$

This expression vanishes automatically for $M_{1}^{(0)}=m=0$. We prefer to measure dimensionful quantities in terms of $1 / a_{s}$ rather than $1 / a_{t}$. When exploring $\chi \geq 1$ it makes more sense to fix $a_{s}$ and let $a_{t}$ be arbitrarily fine, rather than to fix $a_{t}$ and let $a_{s}$ become arbitrarily coarse. In the isotropic limit (40) agrees with formulas in the literature [5, [3].

\section{Wave Function Renormalization}

In order to extract a general formula for the wave function renormalization $Z_{2}$ we need to find the residue of $\bar{G}\left(p_{0}, \vec{p}=0\right)$ at the pole $p_{0}=i M_{1}$. In terms of the variable $z$ the Fourier transform in (15) has the form

$$
\oint_{|z|=1} \frac{d z}{(2 \pi i) z}(z)^{t / a_{t}} \frac{g(z)}{f(z)}
$$


where the integral is taken over the unit circle. To find the residue we expand the denominator around $z_{1}=e^{-a_{t} M_{1}}$

$$
f(z)=\left(z-z_{1}\right)\left(\frac{d f}{d z}\right)_{z=z_{1}}+\cdots .
$$

The contribution from the physical pole to $G(t, 0)$ is then

$$
e^{-M_{1} t}\left(\frac{g(z)}{z f^{\prime}(z)}\right)_{z=z_{1}}
$$

One finds for the numerator

$$
g\left(z=z_{1}\right)=\left(\gamma_{0}+1\right)\left(1-B_{0}\left(i M_{1}, m\right)\right) \sinh \left(a_{t} M_{1}\right)
$$

and for the denominator

$$
\begin{aligned}
& 2\left(1-B_{0}\left(i M_{1}, m\right)\right) \sinh \left(a_{t} M_{1}\right) \times \\
& \left\{\chi \sinh \left(a_{t} M_{1}\right)+\cosh \left(a_{t} M_{1}\right)+\left(i \frac{d}{d\left(a_{t} p_{0}\right)}\left[i B_{0}\left(p_{0}, m\right) \sin \left(a_{t} p_{0}\right)+C\left(p_{0}, m\right)\right]\right)_{p_{0}=i M_{1}}\right\}
\end{aligned}
$$

using

$$
\left(z \frac{d f}{d z}\right)_{z=z_{1}}=-i\left(\frac{d f}{d\left(a_{t} p_{0}\right)}\right)_{p_{0}=i M_{1}}
$$

One can now read off $Z_{2}$ and after recognizing the last term in (45) as derivatives acting on different parts of $a_{t} \Sigma\left(p_{0}, \vec{p}=0, m\right)$, one obtains

$$
Z_{2}^{-1}=\chi \sinh \left(a_{t} M_{1}\right)+\cosh \left(a_{t} M_{1}\right)+i \operatorname{tr}\left(\frac{\left(\gamma_{0}+1\right)}{4} \frac{d}{d p_{0}} \Sigma\left(p_{0}, \vec{p}=0, m\right)\right)_{p_{0}=i M_{1}}
$$

The one-loop approximation to $Z_{2}$ is obtained by expanding $M_{1}$ once again in $\alpha_{s}$.

$$
\begin{aligned}
Z_{2}^{-1}= & \chi \sinh \left(a_{t} M_{1}^{(0)}\right)+\cosh \left(a_{t} M_{1}^{(0)}\right)+\alpha_{s} a_{t} M_{1, s u b}^{(1)}\left(\chi \cosh \left(a_{t} M_{1}^{(0)}\right)+\sinh \left(a_{t} M_{1}^{(0)}\right)\right) \\
& +i \operatorname{tr}\left(\frac{\left(\gamma_{0}+1\right)}{4} \frac{d}{d p_{0}} \Sigma\left(p_{0}, \vec{p}=0, m\right)\right)_{p_{0}=i M_{1}^{(0)}} \\
= & Z_{2}^{(0)-1}\left[1+\frac{\alpha_{s}}{\chi} a_{s} M_{1, s u b}^{(1)}\left(\chi \cosh \left(a_{t} M_{1}^{(0)}\right)+\sinh \left(a_{t} M_{1}^{(0)}\right)\right) Z_{2}^{(0)}\right. \\
& \left.+i \operatorname{tr}\left(\frac{\left(\gamma_{0}+1\right)}{4} \frac{d}{d p_{0}} \Sigma\left(p_{0}, \vec{p}=0, m\right)\right)_{p_{0}=i M_{1}^{(0)}} Z_{2}^{(0)}\right]+O\left(\alpha_{s}^{2}\right) .
\end{aligned}
$$

In the last expression we have found it convenient to factor out the tree-level $Z_{2}^{(0)-1}$. Equations (47) and (48) go over into the formulas of [5] in the isotropic limit. 


\section{Speed of Light Renormalization}

In order to discuss renormalization of the speed of light one needs to look at the inverse momentum space propagator at small but nonzero spatial momentum.

$$
\begin{aligned}
a_{t} \bar{G}^{-1}(p)= & a_{t} \bar{G}_{0}^{-1}(p)-a_{t} \Sigma(p) \\
= & i \gamma_{0}\left(1-B_{0}\right) \sin \left(a_{t} p_{0}\right)+i \frac{1}{\chi} \sum_{j}\left[\gamma_{j}\left(C_{0} K_{j}-B_{j}\right) \sin \left(a_{s} p_{j}\right)\right] \\
& +a_{t} m+\chi-\chi \cos \left(a_{t} p_{0}\right)-C,
\end{aligned}
$$

with $K_{j}=1$ for $\mathcal{S}_{\text {clover }}$ and $K_{j}=\left(4-\cos \left(a_{s} p_{j}\right)\right) / 3$ for $\mathcal{S}_{D 234}^{I, I I}$. One can rewrite $\bar{G}^{-1}(p)$ as

$$
\begin{aligned}
a_{t} \bar{G}^{-1}(p)= & \left(1-B_{0}\right)\left\{i \gamma_{0} \sin \left(a_{t} p_{0}\right)+i \frac{1}{\chi} \sum_{j}\left[\gamma_{j} \frac{\left(C_{0} K_{j}-B_{j}\right)}{\left(1-B_{0}\right)} \sin \left(a_{s} p_{j}\right)\right]\right\} \\
& +a_{t} m+\chi-\chi \cos \left(a_{t} p_{0}\right)-C .
\end{aligned}
$$

$C_{0}$ is adjusted so that for small $a_{s} p_{j}$ the relative coefficient of the $\gamma_{0} \sin \left(a_{t} p_{0}\right)$ and the $\gamma_{j} a_{s} p_{j} / \chi$ terms remains equal to unity. $\left(C_{0} K_{j}-B_{j}\right) \sin \left(a_{s} p_{j}\right)=\left(C_{0}-B_{j}\right) a_{s} p_{j}$ for all quark actions in this limit (of course, $K_{j} \sin \left(a_{s} p_{j}\right)$ is a better approximation to the continuum $a_{s} p_{j}$ in the D234 action than in the clover action), and one has

$$
C_{0}=1+B_{j}\left(C_{0}\right)-B_{0}\left(C_{0}\right) \approx 1+B_{j}\left(C_{0}=1\right)-B_{0}\left(C_{0}=1\right)+O\left(\alpha_{s}^{2}\right)
$$

Just as with $Z_{2}$ we will define the speed of light renormalization at the zero spatial momentum mass shell point $p=\left(i M_{1}, \overrightarrow{0}\right)$. From (27) the two terms $B_{j}$ and $B_{0}$ needed for $C_{0}$ at one-loop can be extracted through

$$
\begin{array}{rlr}
B_{j} & =-i \frac{\chi}{4} \operatorname{tr}\left(\gamma_{j} \frac{\partial}{\partial\left(a_{s} p_{j}\right)} a_{t} \Sigma(p)\right)_{p=\left(i M_{1}^{(0)}, \overrightarrow{0}\right)}, \\
B_{0} & =-\frac{1}{4} \frac{\operatorname{tr}\left(\gamma_{0} a_{t} \Sigma\left(i M_{1}^{(0)}, \overrightarrow{0}\right)\right)}{\sinh \left(a_{t} M_{1}^{(0)}\right)} \quad m>0 \\
& \text { or } \quad m=0 . \\
& =-\frac{i}{4} \operatorname{tr}\left(\gamma_{0} \frac{\partial}{\partial p_{0}} \Sigma(p)\right)_{p=(0, \overrightarrow{0})} & m
\end{array}
$$

We note that in the massive case there is nontrivial renormalization of $C_{0}$ even in the isotropic limit $\chi=1$, due to our noncovariant mass shell condition, $p=\left(i M_{1}, \overrightarrow{0}\right)$. Nevertheless, we believe the above definition of the renormalization of the speed of light is a sensible and physical one.

\section{ONE-LOOP CONTRIBUTIONS TO $\Sigma(P)$}

In the previous section one-loop corrections for $M_{1}, Z_{2}$ and $C_{0}$ were determined in terms of traces over $\Sigma(p)$ or over derivatives acting on $\Sigma(p)$. In this section we describe the lattice 
perturbation theory diagrams that contribute to $\Sigma(p)$ at one loop. For all quark actions considered one can write

$$
\Sigma(p)=\Sigma^{r e g}(p)+\Sigma^{t a d}(p)+\Sigma^{t . i \cdot}(p) .
$$

$\Sigma^{r e g}$ is the regular "rainbow" diagram, the only diagram that exists in a continuum self energy calculation. $\Sigma^{\text {tad }}$ denotes contributions from the lattice artifact tadpole diagram and $\Sigma^{t . i .}$ comes from perturbatively expanding the $u_{s}$ 's and $u_{t}$ 's entering definitions of tadpole improved derivatives (see Appendix). The main idea behind tadpole-improved perturbation theory [8] is to have $\Sigma^{t . i}$. cancel the bulk of $\Sigma^{t a d}$. We find in many instances, especially with $\mathcal{S}_{\text {clover }}$, that cancellation is complete if one uses the Landau link definition for $u_{s}$ and $u_{t}$ and works in Landau gauge.

\section{A. $\Sigma^{r e g}(p)$}

In terms of the gauge propagator $D_{\mu \nu}$, quark propagator $\bar{G}_{0}$ and the vertex functions $V_{\mu}$, one can write $a_{t} \Sigma^{r e g}(p)$ as the following loop integral over the dimensionless momentum variables $-\pi \leq k_{\mu} \leq \pi$.

$$
\begin{aligned}
& a_{t} \Sigma^{r e g}(p) \\
& =g^{2} \frac{4}{3} \sum_{\mu, \nu} \int \frac{d^{4} k}{(2 \pi)^{4}}\left\{V_{\mu}(a p, a p-k) \frac{\bar{G}_{0}(a p-k)}{a_{t}} V_{\nu}(a p-k, a p)\right\} D_{\mu \nu}\left(k, \alpha_{g}\right) \\
& =g^{2} \frac{4}{3} \sum_{\mu, \nu} \int \frac{d^{4} k}{(2 \pi)^{4}}\left\{V_{\mu}(a p, a p-k)[-i \gamma \cdot K \sin +\Omega]_{(a p-k)} V_{\nu}(a p-k, a p)\right\} \frac{D_{\mu \nu}\left(k, \alpha_{g}\right)}{\left(K^{2} \sin ^{2}+\Omega^{2}\right)_{(a p-k)}}
\end{aligned}
$$

where, ap stands for $\left(a_{t} p_{0}, a_{s} \vec{p}\right)$ and $K \sin$ for $K_{0} \sin \left((a p-k)_{0}\right)$ or for $K_{j} \sin \left((a p-k)_{j}\right) / \chi$. $K_{\mu}(k), \Omega(k), V_{\mu}\left(k^{\prime}, k\right)$ and $D_{\mu \nu}\left(k, \alpha_{g}\right)$ are detailed in the Appendix. The argument $\alpha_{g}$ in the gauge propagator comes from the gauge fixing term, with $\alpha_{g}=1$ and $\alpha_{g}=0$ corresponding to Feynman and Landau gauges respectively. Our codes have been written for general $\alpha_{g}$ and we have used gauge invariance of $M_{1}$ and $C_{0}$ as one check on our results.

Equation (56) has the familiar form for a self energy integral. The only subtlety is to verify that one is indeed calculating $\Sigma^{r e g}$ measured in units of $1 / a_{t}$, given the conventions in our Feynman rules. As explained in the Appendix, we choose to work with a dimensionless momentum space gauge propagator $D_{\mu \nu}$. It comes from the Fourier transform of the dimensionless correlator $\left\langle\left(a_{\mu} A_{\mu}\right)\left(a_{\nu} A_{\nu}\right)\right\rangle$. The relation between $D_{\mu \nu}$ and a more conventional propagator of dimension $1 /(\text { energy })^{2}$, denoted $\tilde{D}_{\mu \nu}$, is

$$
D_{\mu \nu}=\frac{a_{\mu} a_{\nu}}{a_{s}^{3} a_{t}} \tilde{D}_{\mu \nu}
$$

Our vertex functions, $V_{\mu}$, are also subtlely different from those in isotropic lattice perturbation theory. They keep tract of the $1 / a_{\mu}$ in the derivatives, i.e. of whether one has a $1 / a_{s}$ or $1 / a_{t}$ there. If $\tilde{V}_{\mu}$ are vertex functions normalized such that $\tilde{V}_{\mu} \rightarrow-i \gamma_{\mu}$ for all $\mu$ in the continuum limit, then the relation to the $V_{\mu}$ of (56) is given by 


$$
V_{\mu}=\frac{a_{t}}{a_{\mu}} \tilde{V}_{\mu}
$$

Using (57) and (58) one can write

$$
\begin{aligned}
a_{t} \Sigma^{r e g}(p) & =g^{2} \frac{4}{3} \sum_{\mu, \nu} \int \frac{d^{4} k}{(2 \pi)^{4}}\left\{\left(\frac{a_{t}}{a_{\mu}} \tilde{V}_{\mu}\right) \frac{\bar{G}_{0}}{a_{t}}\left(\frac{a_{t}}{a_{\nu}} \tilde{V}_{\nu}\right)\right\}\left(\frac{a_{\mu} a_{\nu}}{a_{s}^{3} a_{t}} \tilde{D}_{\mu \nu}\right) \\
& =a_{t} g^{2} \frac{4}{3} \sum_{\mu, \nu} \int \frac{d^{4} k}{(2 \pi)^{4} a_{s}^{3} a_{t}}\left\{\tilde{V}_{\mu} \bar{G}_{0} \tilde{V}_{\nu}\right\} \tilde{D}_{\mu \nu} .
\end{aligned}
$$

To evaluate (56) we made extensive use of the symbolic manipulation package Mathematica. The integrals themselves were done using the VEGAS program [16]. The various steps involving Mathematica were to 1 . calculate the products $V_{\mu}[-i \gamma \cdot K \sin +\Omega] V_{\nu} ; 2$. carry out the Dirac traces such as $\operatorname{tr}\left\{\left(1+\gamma_{0}\right) \Sigma\right\} ; 3$. take derivatives with respect to external momenta ; 4. put things on the mass shell $p=\left(i M_{1}, \overrightarrow{0}\right)$; and 5 . use trigonometric identities to re-express the full integrands in (56) in terms of powers of $\hat{k}_{\mu} \equiv 2 \sin \left(k_{\mu} / 2\right)$. The last step facilitated speedy evaluation of the integrand by VEGAS.

Both $M_{1}$ and $C_{0}$ are physical quantities. In addition to being gauge invariant they are also IR finite. The wave function renormalization $Z_{2}$, on the other hand, is gauge dependent and also generally logarithmically IR divergent. In any calculation of a physical quantity this IR divergence will eventually be cancelled by vertex corrections and/or matching to continuum operators. In this article we will isolate the gauge dependent IR divergence in $Z_{2}$, verify that it is the same as in the corresponding continuum theory and present results for the remaining IR finite parts. The IR divergence is found in the contribution from $\Sigma^{\text {reg }}$ to $Z_{2}$. More specifically it resides in the following term in (48)

$$
i \operatorname{tr}\left(\frac{\left(\gamma_{0}+1\right)}{4} \frac{d}{d p_{0}} \Sigma^{r e g}\left(p_{0}, \vec{p}=0, m\right)\right)_{p_{0}=i M_{1}^{(0)}} Z_{2}^{(0)} .
$$

We adopt the method of reference [13] to subtract IR divergent contributions inside integrands and rewrite (60) as

$$
\begin{aligned}
& \int_{k}\left\{\sum_{\mu, \nu} i \operatorname{tr}\left(\frac{\left(\gamma_{0}+1\right)}{4} \frac{d}{d p_{0}}\left[V_{\mu} \frac{\bar{G}_{0}}{a_{t}} V_{\nu}\right]_{p_{0}=i M_{1}^{(0)}} D_{\mu \nu} Z_{2}^{(0)}\right)-\mathcal{F}_{\text {sub }}\left(k, m_{\text {eff }}, \Lambda, \lambda\right)\right\} \\
& \quad+F\left(m_{\text {eff }}, \Lambda, \lambda\right),
\end{aligned}
$$

with

$$
F\left(m_{e f f}, \Lambda, \lambda\right)=\int_{k} \mathcal{F}_{\text {sub }}\left(k, m_{\text {eff }}, \Lambda, \lambda\right)
$$

and

$$
\int_{k} \equiv g^{2} \frac{4}{3} \int \frac{d^{4} k}{(2 \pi)^{4}}
$$


Explicit forms for $\mathcal{F}_{\text {sub }}\left(k, m_{\text {eff }}, \Lambda, \lambda\right)$ and $F\left(m_{\text {eff }}, \Lambda, \lambda\right)$ are given in the Appendix. $\lambda$ is a gluon mass introduced to regulate IR divengences. $\mathcal{F}_{\text {sub }}$ has been constructed to match the same IR divergence as the first term inside the integral in (61). As a result the integral becomes independent of $\lambda$. The other condition on $\mathcal{F}_{\text {sub }}$ is that the integral (62) be easy to do analytically. The simplest approach is to use a continuum self energy expression for $\mathcal{F}_{\text {sub }}$ with an appropriate choice for the mass parameter $m_{\text {eff }}$. The need to adjust $m_{\text {eff }}$ to optimize matching of the IR behaviours in $\mathcal{F}_{\text {sub }}$ and the lattice integrand, was emphasized in reference 13 and following that work we find

$$
a_{t} m_{e f f}=\sinh \left(a_{t} M_{1}^{(0)}\right) \frac{\cosh \left(a_{t} M_{1}^{(0)}\right)+\chi \sinh \left(a_{t} M_{1}^{(0)}\right)}{1+\chi \sinh \left(a_{t} M_{1}^{(0)}\right)} .
$$

The same $\mathcal{F}_{\text {sub }}$ and $m_{\text {eff }}$ work for both the clover and D234 quark actions since the IR structure of the two theories agree. Finally, $\Lambda \leq \pi$ in the above expressions is a cutoff imposed on $\mathcal{F}_{\text {sub }}$ so that $\mathcal{F}_{\text {sub }}=0$ for $k^{2}>\Lambda^{2}$. The full expression (61) must be independent of $\Lambda$.

\section{B. $\Sigma^{\operatorname{tad}}(p)$}

The second contribution to $\Sigma(p)$ is the tadpole contribution $\Sigma^{t a d}(p)$ coming from the two-gluon emission vertices listed in the Appendix. For quark action $\mathcal{S}_{D 234}^{I I}$ one has

$$
\begin{aligned}
a_{t} \Sigma^{t a d}(p)= & \frac{1}{2}\left[i \gamma_{0} \sin \left(a_{t} p_{0}\right)-\chi \cos \left(a_{t} p_{0}\right)\right] \int_{k} D_{00} \\
& +\frac{1}{2 \chi} \frac{1}{3} \sum_{j} \int_{k} D_{j j}\left\{i \gamma_{j}\left[\left(3+C_{3}\right) \sin \left(a_{s} p_{j}\right)-2 C_{3} \sin \left(2 a_{s} p_{j}\right) \cos \left(\frac{k_{j}}{2}\right)\right]\right. \\
& \left.-\left[\left(3+C_{4}\right) \cos \left(a_{s} p_{j}\right)-C_{4} \cos \left(2 a_{s} p_{j}\right) \cos ^{2}\left(\frac{k_{j}}{2}\right)\right]\right\} .
\end{aligned}
$$

$\Sigma^{t a d}(p)$ in the case of $\mathcal{S}_{\text {clover }}$ is obtained by setting $C_{3}=C_{4}=0$ in the above expression. The appropriate traces and derivatives with respect to external momenta can be carried out immediately and one has

$$
\begin{aligned}
& -\operatorname{tr}\left\{\frac{\left(\gamma_{0}+1\right)}{4} a_{t} \Sigma^{\text {tad }}\right\}_{p=\left(i M_{1}^{(0)}, \overrightarrow{0}\right)} \\
& =\left\{\begin{array}{lc}
\frac{1}{2}\left[\sinh \left(a_{t} M_{1}^{(0)}\right)+\chi \cosh \left(a_{t} M_{1}^{(0)}\right)\right] \int_{k} D_{00}+\frac{1}{2 \chi} \sum_{j} \int_{k} D_{j j} & \mathcal{S}_{\text {clover }} \\
\frac{1}{2}\left[\sinh \left(a_{t} M_{1}^{(0)}\right)+\chi \cosh \left(a_{t} M_{1}^{(0)}\right)\right] \int_{k} D_{00}+\frac{1}{6 \chi} \sum_{j} \int_{k} D_{j j}\left[4-\cos ^{2}\left(\frac{k_{j}}{2}\right)\right] & \mathcal{S}_{D 234}^{I I}
\end{array}\right. \\
& i \operatorname{tr}\left\{\frac{\left(\gamma_{0}+1\right)}{4} \frac{d}{d p_{0}} \Sigma^{\operatorname{tad}}\right\}_{p=\left(i M_{1}^{(0)}, \overrightarrow{0}\right)}=-\frac{1}{2}\left[\cosh \left(a_{t} M_{1}^{(0)}\right)+\chi \sinh \left(a_{t} M_{1}^{(0)}\right)\right] \int_{k} D_{00}
\end{aligned}
$$




$$
B_{j}^{\text {tad }}-B_{0}^{\text {tad }}= \begin{cases}\frac{1}{2} \int_{k} D_{j j}-\frac{1}{2} \int_{k} D_{00} & \mathcal{S}_{\text {clover }} \\ \frac{2}{3} \int_{k} D_{j j} \sin ^{2}\left(\frac{k_{j}}{2}\right)-\frac{1}{2} \int_{k} D_{00} & \mathcal{S}_{D 234}^{I I}\end{cases}
$$

where $B_{j}^{\text {tad }}$ and $B_{0}^{\text {tad }}$ are the contributions from the tadpole diagram to (52) and (53) or (54). All the integrals are IR finite and very easy to carry out numerically. Contributions from $\Sigma^{\text {tad }}$ typically dominate over those from $\Sigma^{r e g}$ but the bulk if not all of it is cancelled by $\Sigma^{t . i .}$.

\section{C. $\Sigma^{t . i \cdot}(p)$}

The lattice covariant derivatives in the quark actions are tadpole-improved. They are listed in the Appendix. In momentum space one has, for instance

$$
\nabla_{\mu} \rightarrow i \sin \left(a_{\mu} p_{\mu}\right) / u_{\mu} \approx i \sin \left(a_{\mu} p_{\mu}\right)\left[1+\alpha_{s} u_{\mu}^{(2)}\right]+O\left(\alpha_{s}^{2}\right)
$$

where we have perturbatively expanded

$$
u_{\mu}=1-\alpha_{s} u_{\mu}^{(2)}+O\left(\alpha_{s}^{2}\right)
$$

Even in the absence of the regular and tadpole one-loop diagrams there are hence $O\left(\alpha_{s}\right)$ terms in the quark propagator. We denote the inverse quark propagator with the $u_{\mu}$ 's still in place as $\bar{G}_{0, u 0}^{-1}(p)$, so that $\bar{G}_{0}^{-1}(p) \equiv \bar{G}_{0, u 0=1}^{-1}(p)$. Through $O\left(\alpha_{s}\right)$ eqn.(11) can be written as

$$
\bar{G}^{-1}=\bar{G}_{0, u 0}^{-1}-\Sigma^{r e g}-\Sigma^{t a d} \equiv \bar{G}_{0}^{-1}-\Sigma^{r e g}-\Sigma^{t a d}-\Sigma^{t . i .}
$$

or

$$
\Sigma^{t . i .}=\bar{G}_{0, u 0=1}^{-1}-\bar{G}_{0, u 0}^{-1} .
$$

From the difference in (72) one sees that one link hops bring in factors of $1-1 / u_{\mu} \approx-\alpha_{s} u_{\mu}^{(2)}$ and two link hops factors of $1-1 / u_{\mu}^{2} \approx-2 \alpha_{s} u_{\mu}^{(2)}$ etc. Using these rules one finds

$$
\begin{aligned}
& a_{t} \Sigma^{t . i .}(p)= \alpha_{s} u_{t}^{(2)}\left[-i \gamma_{0} \sin \left(a_{t} p_{0}\right)+\chi \cos \left(a_{t} p_{0}\right)\right]+ \\
& \alpha_{s} u_{s}^{(2)} \frac{1}{3 \chi} \sum_{j}\left\{-i \gamma_{j}\left[\left(3+C_{3}\right) \sin \left(a_{s} p_{j}\right)-C_{3} \sin \left(2 a_{s} p_{j}\right)\right]\right. \\
&\left.+\quad\left[\left(3+C_{4}\right) \cos \left(a_{s} p_{j}\right)-\frac{C_{4}}{2} \cos \left(2 a_{s} p_{j}\right)\right]\right\} .
\end{aligned}
$$

The relevant traces and derivatives become 


$$
\begin{aligned}
& -\operatorname{tr}\left\{\frac{\left(\gamma_{0}+1\right)}{4} a_{t} \Sigma^{t . i .}\right\}_{p=\left(i M_{1}^{(0)}, \overrightarrow{0}\right)} \\
& = \begin{cases}-\left[\sinh \left(a_{t} M_{1}^{(0)}\right)+\chi \cosh \left(a_{t} M_{1}^{(0)}\right)\right] \alpha_{s} u_{t}^{(2)}-\frac{3}{\chi} \alpha_{s} u_{s}^{(2)} & \mathcal{S}_{\text {clover }} \\
-\left[\sinh \left(a_{t} M_{1}^{(0)}\right)+\chi \cosh \left(a_{t} M_{1}^{(0)}\right)\right] \alpha_{s} u_{t}^{(2)}-\frac{1}{\chi} \frac{7}{2} \alpha_{s} u_{s}^{(2)} & \mathcal{S}_{D 234}^{I I}\end{cases} \\
& i \operatorname{tr}\left\{\frac{\left(\gamma_{0}+1\right)}{4} \frac{d}{d p_{0}} \Sigma^{t . i .}\right\}_{p=\left(i M_{1}^{(0)}, \overrightarrow{0}\right)}=\left[\cosh \left(a_{t} M_{1}^{(0)}\right)+\chi \sinh \left(a_{t} M_{1}^{(0)}\right)\right] \alpha_{s} u_{t}^{(2)} \\
& B_{j}^{t . i .}-B_{0}^{t . i .}= \begin{cases}\alpha_{s}\left(u_{t}^{(2)}-u_{s}^{(2)}\right) & \mathcal{S}_{\text {clover }} \& \mathcal{S}_{D 234}^{I I} \\
\alpha_{s}\left(u_{t}^{(2)}-\frac{2}{3} u_{s}^{(2)}\right) & \mathcal{S}_{\text {clover }}\end{cases}
\end{aligned}
$$

The Landau mean link definition of $u_{\mu}$ is given by

$$
u_{\mu} \equiv\left\langle\frac{1}{3} \operatorname{Tr} U_{\mu}\right\rangle_{\alpha_{g}=0} \approx 1-\alpha_{s} u_{\mu}^{(2)}=1-\frac{1}{2} \int_{k} D_{\mu \mu}\left(\alpha_{g}=0\right) .
$$

If one evaluates $\Sigma^{\text {tad }}$ in Landau gauge then (66) \& (74), (67) \& (75) and (68) \& (76) cancel for $\mathcal{S}_{\text {clover }}$. (67) \& (75) also cancel for $\mathcal{S}_{D 234}^{I I}$ and for the other two traces cancellation is almost complete. The difference between contributions from $\Sigma^{t a d}$ and $\Sigma^{t . i .}$ would go away if one replaces $\cos ^{2}(k / 2)$ and $\sin ^{2}(k / 2)$ by their averages $1 / 2$. Hence, it is easy to see in this calculation how tadpole improving terms in the lattice action eliminates lattice artifact contributions in perturbation theory.

\section{RESULTS}

In this section we summarize results for the one-loop coefficients, $a_{s} M_{1, s u b}^{(1)}, Z_{2}^{(1)}$ and $C_{0}^{(1)}$ for mass, wave function and speed of light renormalizations respectively. These follow from equations (40), (48) and (51) - (54) and each has, as explained in the previous section, contributions from regular, tadpole and t.i. diagrams. The numbers in our Tables are coefficients of $\alpha_{s}$. The Landau mean link definition of $u_{\mu}$ is used throughout to implement tadpole improvement.

$$
\text { A. } a_{s} M_{1, s u b}^{(1)}
$$

In Table II we present results for $a_{s} M_{1, s u b}^{(1)}$ for action $\mathcal{S}^{A}$ for several values of $a_{s} M_{1}^{(0)}$. We list separately contributions from $\Sigma^{r e g}, \Sigma^{\text {tad }}$ and $\Sigma^{t . i .}$. The fourth column gives the gauge invariant combination $(\mathrm{reg}+\mathrm{tad})$ and the sixth column gives $a_{s} M_{1, \text { nosub }}^{(1)} \equiv(\mathrm{reg}+\mathrm{tad}+$ 
t.i.), the full tadpole improved one-loop correction before subtraction. Carrying out the subtraction according to (40), one obtains $a_{s} M_{1, \text { sub }}^{(1)}$ which is given in the last column

$$
a_{s} M_{1, \text { sub }}^{(1)}=a_{s} M_{1, \text { nosub }}^{(1)}-\frac{a_{s} M_{1, \text { nosub }}^{(1)}(0)}{\chi \sinh \left(a_{t} M_{1}^{(0)}\right)+\cosh \left(a_{t} M_{1}^{(0)}\right)} .
$$

All our calculations have been carried out for two values of the gauge fixing parameter $\alpha_{g}$, 1.0 and 0.0. Table II lists both sets of results and one sees that gauge invariant quantities are independent of $\alpha_{g}$ within numerical integration errors ( which we take to be at the \pm 0.003 to \pm 0.006 level depending on the mass). Our results for $a_{s} M_{1}^{(0)}=0$ agree with those from [12].

We plot $a_{s} M_{1, \text { sub }}^{(1)}$ versus $a_{s} M_{1}^{(0)}$ in Fig. 1 . One sees that the mass dependence is smooth and that one reaches saturation rapidly already around $a_{s} M_{1}^{(0)} \sim 3.0-5.0$. We also compare with non-tadpole improved results for which the curve saturates around 1.827 rather than around 1.077. In considering the large mass limit it is useful to note that the factor $Z_{2}^{(0)}=$ $1 /\left[\chi \sinh \left(a_{t} M_{1}^{(0)}\right)+\cosh \left(a_{t} M_{1}^{(0)}\right)\right]$ appearing in (40) and (48) vanishes exponentially in this limit. The only terms that survive into the static limit are those where $Z_{2}^{(0)}$ is multiplied by an exponentially increasing function of $a_{s} M_{1}^{(0)}$. It is easy to see, for instance, that the subtraction term in (78) or the spatial tadpoles in (66) become irrelevant in the static limit. Furthermore $a_{s} M_{1, \text { sub }}^{(1)}$ becomes identical for $\mathcal{S}_{\text {Wilson }}, \mathcal{S}_{\text {clover }}$ and $\mathcal{S}_{D 234}^{I I}$ in this limit and the only difference between the current calculations and those of reference [17] resides in the glue action (we have verified that by switching to the unimproved Wilson glue action results of [17] are reproduced).

In Tables III, IV and V we summarize results for $a_{s} M_{1, s u b}^{(1)}$ for the other actions listed in Table I. We also list the combination (reg + tad) for each case. If one chooses to implement tadpole improvement differently from what we have done here or decides not to tadpole improve, then $a_{s} M_{1, s u b}^{(1)}$ can be calculated straightforwardly from $(\mathrm{reg}+\mathrm{tad})$ and the formulas presented in this paper. For action $\mathcal{S}^{C}$ we list only massless results for reasons explained in section II. The dispersion relations of this and similar actions are discussed in reference [18]. Our choices for anisotropy values in Tables IV and V, were dictated in part with an eye towards practical numerical simulations. Values such as $\chi=3.6$ and $\chi=5.3$ were taken from recent work on nonperturbative determinations of the renormalized anisotropy in pure glue theory [19]. For one value $a_{s} M_{1}^{(0)}=1$ we plot $a_{s} M_{1, s u b}^{(1)}$ versus $\chi$ in Fig. 2 using the action $\mathcal{S}^{D}$. From Tables IV and V and from Fig. 2 one sees that the dependence of $a_{s} M_{1, s u b}^{(1)}$ on $\chi$ is very mild. Effects of tadpole improvement are significant only for small values of $\chi$. This is because due to cancellations in the subtraction of equation (78) only the temporal tadpole and the temporal Landau link term $u_{t}^{(2)}$ contribute to $a_{s} M_{1, s u b}^{(1)}$ and both these become small as $\chi$ increases.

Finally we mention that the one-loop expression for the critical bare mass $m_{c}$ is given by

$$
a_{t} m_{c}=-\frac{\alpha_{s}}{\chi} a_{s} M_{1, \text { nosub }}^{(1)}(0)+O\left(\alpha_{s}^{2}\right) .
$$




\section{B. $Z_{2}^{(1)}$}

Starting with (48) we define

$$
Z_{2}=Z_{2}^{(0)}\left[1+\alpha_{s}\left(Z_{2}^{(1)}+Z_{2}^{(1) I R}\right)+O\left(\alpha_{s}^{2}\right)\right]
$$

with

$$
Z_{2}^{(1) I R}= \begin{cases}\frac{1}{3 \pi}\left[1+\left(\alpha_{g}-1\right)\right] \ln \left(\lambda^{2}\right) & m=0 \\ \frac{1}{3 \pi}\left[-2+\left(\alpha_{g}-1\right)\right] \ln \left(\lambda^{2}\right) & m>0\end{cases}
$$

$\lambda$ is the gluon mass in units of $1 / a_{s}$. It is the coefficient of $\alpha_{s}$ after factoring out $Z_{2}^{(0)}$ that has the same IR $\ln (\lambda)$ and $\ln (a m)$ structure as the continuum wave function renormalization constant. From (48) one also sees that there are two contributions to $Z_{2}^{(1)}$, one coming from the $d / d p_{0}$ derivative term and the second from the expansion of $M_{1}$. Accordingly we write

$$
Z_{2}^{(1)}=Z_{2, d p_{0}}^{(1)}+Z_{2, M_{1}}^{(1)}
$$

In the literature $Z_{2, M_{1}}^{(1)}$ is not always included as part of the definition of $Z_{2}^{(1)}$. $Z_{2, d p_{0}}^{(1)}$ alone with unimproved Wilson glue goes over in the large mass limit to the wave function renormalization of reference [17]. Including $Z_{2, M_{1}}^{(1)}$ leads to the static result of reference [20] which has been used in many subsequent static calculations, for instance in [21]. This latter static value also corresponds to the large mass limit of the one-loop $Z_{2}$ calculated in many versions of NRQCD actions [22].

Table VI presents results for $Z_{2, d p_{0}}^{(1)}$ and the full $Z_{2}^{(1)}$ for the action $\mathcal{S}^{A}$. Again we agree with reference [12] for $m=0$. However, one notices that the massive data do not tend towards the massless result as $a_{s} M_{1}^{(0)}$ decreases. This is because our massive numbers include $\ln (\mathrm{am})$ contributions which will eventually diverge, whereas in the massless theory we have set the fermion mass identical to zero from the beginning. This leads to different IR structure for the two theories (see Appendix B for some further discussions). In a matching calculation one will be looking at differences between the lattice and continuum $Z_{2}$. As long as IR divergences are handled in the same manner in the lattice and continuum evaluations, one should not run into any problems and the $m \rightarrow 0$ limit should be smooth. For instance, using dimensional regularization in the $\overline{M S}$ scheme one finds in Feynman gauge the UV finite continuum results

$$
Z_{2}^{(1) \text { cont. }}= \begin{cases}\frac{1}{3 \pi}\left[\ln \left(\frac{\hat{\lambda}^{2}}{\mu^{2}}\right)+\frac{1}{2}\right] & m=0 \\ \frac{1}{3 \pi}\left[\ln \left(\frac{m^{2}}{\mu^{2}}\right)+2 \ln \left(\frac{m^{2}}{\hat{\lambda}^{2}}\right)-4\right] & m>0\end{cases}
$$

Taking the difference between continuum and lattice wave function renormalization constants, it makes sense to consider the following subtracted $Z_{2}$ factors.

$$
Z_{2, d i f f}^{(1)}=Z_{2, d p_{0}}^{(1)}- \begin{cases}\frac{1}{3 \pi} \frac{1}{2} & m=0 \\ \frac{1}{3 \pi}\left[3 \ln \left(a_{s} M_{1}^{(0)}\right)^{2}-4\right] & m>0\end{cases}
$$


Numbers for $Z_{2, \text { diff }}^{(1)}$ are given in Table VII and one sees that the $m \rightarrow 0$ behaviour is smooth.

In Tables VIII through XII we present $Z_{2, d p_{0}}^{(1)}$ and $Z_{2}^{(1)}$ for other actions. One does not find any dramatic changes with differing actions and/or anisotropy. The IR subtractions of Appendix B worked well in all actions for $a_{s} M_{1}^{(0)}<\sim 5$. For larger masses VEGAS errors became large especially for $\chi=1$. Hence, we only present results up to $a_{s} M_{1}^{(0)}=5$. For $\chi>1$ problems were less severe in general. In Tables VI - XII the numerical integration errors are at the \pm 0.02 level for $a_{s} M_{1}^{(0)}=5.0$ and $\chi<3$, at the \pm 0.006 level for $a_{s} M_{1}^{(0)}=$ $0.01,0.05$ and 0.10 and at the \pm 0.004 level for all other cases. A more sophisticated method for handling IR divergent integrals appears necessary if accurate results are required for larger masses. Many quantities, however, are close to saturation by the time one reaches $a_{s} M_{1}^{(0)}=5$.

\section{C. $C_{0}^{(1)}$}

For the speed of light renormalization we present the most detailed results for action $\mathcal{S}^{B}$ rather than for $\mathcal{S}^{A}$ since the former, for $\chi>1$, is genuinely anisotropic. In Table XIII we list separately regular and tadpole diagram contributions, their gauge invariant sum $(r e g+t a d) \equiv\left(C_{0}^{(1)}\right.$ no t.i. $)$ and the fully tadpole improved result $\left(C_{0}^{(1)}\right.$ with t.i. $)$, all for action $\mathcal{S}^{B}$ and at fixed anisotropy $\chi=4.0 . C_{0}^{(1)}$ is independent of $\alpha_{g}$ within numerical integration errors which are the most severe when using (53) for nonzero but small masses. In Figure 3. we plot $C_{0}^{(1)}$ both with and without tadpole improvement versus $a_{s} M_{1}^{(0)}$. Table XIV summarizes results for several $\chi$ values with action $\mathcal{S}^{B}$ and in Figure 4 . we plot $C_{0}^{(1)}$ versus $\chi$ for fixed $a_{s} M_{1}^{(0)}=1.0$. One sees that tadpole improvement has significant effect and causes $C_{0}^{(1)}$ to switch sign for $\chi>1$. Among other things this allows for a smooth $\chi \rightarrow 1$ limit.

In Table XV we present results for actions $\mathcal{S}^{A}$ and $\mathcal{S}^{A^{\prime}}$. In these isotropic actions nontrivial $C_{0}$ comes about because our mass-shell condition $p=\left(i M_{1}, \overrightarrow{0}\right)$ distinguishes between spatial and temporal directions once $M_{1}>0$. Table XVI summarizes results for action $\mathcal{S}^{D}$. Here tadpole improvement does not decrease the magnitude of the one-loop correction, however, for a wide range of mass values it is still true that $C_{0}^{(1)}$ switches sign for $\chi>1$ and that the $\chi \rightarrow 1$ limit becomes smoother after tadpole improvement.

\section{SUMMARY}

We have carried out one-loop perturbative renormalization of the fermion rest mass $M_{1}$, wave function renormalization $Z_{2}$ and the speed of light $C_{0}$ for a range of highly improved actions on isotropic and anisotropic lattices. We find that the dependence of the one-loop coefficients on the anisotropy parameter $\chi=a_{s} / a_{t}$ and on the tree-level mass parameter $a_{s} M_{1}^{(0)}$ is mild, especially after tadpole improvement of the actions. Furthermore, none of the coefficients are particularly large. $M_{1}$ and $C_{0}$ exhibit smooth behaviour as one approaches the massless, large mass, $\chi \rightarrow 1$ and large $\chi$ limits. This also holds for $Z_{2}$ if more 
physical combinations such as the difference between continuum and lattice wave function renormalizations are considered. The next stage in our program would be to extend the present calculations to vertex corrections and to matchings between continuum and lattice currents and other multi-fermion operators.

\section{ACKNOWLEDGMENTS}

This research is supported by grants from the US Department of Energy, DE-FG0291ER40690, and from the Graduiertenkolleg. The authors thank Peter Lepage for many useful conversations. S.G. gratefully acknowledges a grant given by the Max Kade Foundation. J.S. thanks Cornell University for its hospitality while the project was being initiated and the theoretical physics group at the University of Glasgow where part of the work was carried out. Support from a UK PPARC Visiting Fellowship PPA/V/S/1997/00666 is gratefully acknowledged.

\section{APPENDIX A: DEFINITIONS AND FEYNMAN RULES}

In this Appendix we summarize definitions for various terms in the lattice actions and present Feynman rules for gauge and quark propagators and for vertex functions.

Covariant Derivatives Acting on Quark Fields

$$
\begin{aligned}
\nabla_{\mu} \Psi(x)= & \frac{1}{2} \frac{1}{u_{\mu}}\left[U_{\mu}(x) \Psi\left(x+a_{\mu}\right)-U_{\mu}^{\dagger}\left(x-a_{\mu}\right) \Psi\left(x-a_{\mu}\right)\right] \\
\nabla_{\mu}^{(2)} \Psi(x)= & \frac{1}{u_{\mu}}\left[U_{\mu}(x) \Psi\left(x+a_{\mu}\right)+U_{\mu}^{\dagger}\left(x-a_{\mu}\right) \Psi\left(x-a_{\mu}\right)\right]-2 \Psi(x) \\
\nabla_{\mu}^{(3)} \Psi(x)= & \frac{1}{2} \frac{1}{u_{\mu}^{2}}\left[U_{\mu}(x) U_{\mu}\left(x+a_{\mu}\right) \Psi\left(x+2 a_{\mu}\right)-U_{\mu}^{\dagger}\left(x-a_{\mu}\right) U_{\mu}^{\dagger}\left(x-2 a_{\mu}\right) \Psi\left(x-2 a_{\mu}\right)\right] \\
& -\frac{1}{u_{\mu}}\left[U_{\mu}(x) \Psi\left(x+a_{\mu}\right)-U_{\mu}^{\dagger}\left(x-a_{\mu}\right) \Psi\left(x-a_{\mu}\right)\right] \\
\nabla_{\mu}^{(4)} \Psi(x)= & \frac{1}{u_{\mu}^{2}}\left[U_{\mu}(x) U_{\mu}\left(x+a_{\mu}\right) \Psi\left(x+2 a_{\mu}\right)+U_{\mu}^{\dagger}\left(x-a_{\mu}\right) U_{\mu}^{\dagger}\left(x-2 a_{\mu}\right) \Psi\left(x-2 a_{\mu}\right)\right] \\
& -4 \frac{1}{u_{\mu}}\left[U_{\mu}(x) \Psi\left(x+a_{\mu}\right)+U_{\mu}^{\dagger}\left(x-a_{\mu}\right) \Psi\left(x-a_{\mu}\right)\right]+6 \Psi(x)
\end{aligned}
$$

Field Strength Tensors

For the unimproved $F_{\mu \nu}$ of the clover action we use

$$
\begin{aligned}
F_{\mu \nu}(x) & =\frac{1}{2 i}\left(\Omega_{\mu \nu}(x)-\Omega_{\mu \nu}^{\dagger}(x)\right), \\
\Omega_{\mu \nu}(x) & =\frac{1}{4 u_{\mu}^{2} u_{\nu}^{2}} \sum_{\{(\alpha, \beta)\}_{\mu \nu}} U_{\alpha}(x) U_{\beta}\left(x+a_{\alpha}\right) U_{-\alpha}\left(x+a_{\alpha}+a_{\beta}\right) U_{-\beta}\left(x+a_{\beta}\right),
\end{aligned}
$$


with $\{(\alpha, \beta)\}_{\mu \nu}=\{(\mu, \nu),(\nu,-\mu),(-\mu,-\nu),(-\nu, \mu)\}$ for $\mu \neq \nu$ and $U_{-\mu}\left(x+a_{\mu}\right) \equiv U_{\mu}^{\dagger}(x)$. The $O\left(a^{2}\right)$ improved field strength tensor of the D234 actions is

$$
\begin{aligned}
& \tilde{F}_{\mu \nu}(x)=\frac{5}{3} F_{\mu \nu}(x) \\
&-\frac{1}{6} {\left[\frac{1}{u_{\mu}^{2}}\left(U_{\mu}(x) F_{\mu \nu}\left(x+a_{\mu}\right) U_{\mu}^{\dagger}(x)+U_{\mu}^{\dagger}\left(x-a_{\mu}\right) F_{\mu \nu}\left(x-a_{\mu}\right) U_{\mu}\left(x-a_{\mu}\right)\right)-(\mu \leftrightarrow \nu)\right] } \\
&+\frac{1}{6}\left(\frac{1}{u_{\mu}^{2}}+\frac{1}{u_{\nu}^{2}}-2\right) F_{\mu \nu}(x) .
\end{aligned}
$$

The last term ensures that factors of $1 / u_{\mu}$ are correctly removed from those contributions to $U F_{\mu \nu} U^{\dagger}$ and $U^{\dagger} F_{\mu \nu} U$ that end up being four link objects rather than six link ones. In a one-loop calculation, however, one can set $u_{\mu}=1$ everywhere in the definition of the field strength tensor and this correction term is irrelevant.

Both the above covariant derivatives and the field strength tensor are dimensionless. Factors of $1 / a_{t}$ and $1 / a_{s}$ are inserted explicitly where necessary such as in (6).

\section{Gauge Propagator}

The isotropic Symanzik improved gauge action has been discussed quite extensively in the literature [9]. Here we summarize formulas for the anisotropic generalization. We start from the gauge actions $\mathcal{S}_{G}^{I}$ or $\mathcal{S}_{G}^{I I}$ and add to it a gauge fixing term

$$
\begin{aligned}
\mathcal{S}_{g f} & =\frac{1}{2 \alpha_{g}} a_{s}^{3} a_{t} \sum_{x}\left[\frac{1}{a_{t}} \partial_{t} A_{t}+\frac{1}{a_{s}} \sum_{j} \partial_{j} A_{j}\right]^{2} \\
& =\frac{1}{2 \alpha_{g}} \frac{1}{\chi} \sum_{x}\left[\chi^{2} \partial_{t}\left(a_{t} A_{t}\right)+\sum_{j} \partial_{j}\left(a_{s} A_{j}\right)\right]^{2},
\end{aligned}
$$

with $\partial_{\mu} A_{\mu}(x) \equiv A_{\mu}\left(x+a_{\mu} / 2\right)-A_{\mu}\left(x-a_{\mu} / 2\right)$. Equation (A8) expresses $\mathcal{S}_{g f}$ in terms of dimensionless gauge fields $a_{\mu} A_{\mu}$. It is convenient to do so, especially since $\mathcal{S}_{G}^{I, I I}$ are already in dimensionless form with factors of $\chi$ and $1 / \chi$ properly put in place. If $\bar{A}_{\mu}(k)$ is the Fourier transform of $\left(a_{\mu} A_{\mu}\right)$, the quadratic terms in the gauge action become

$$
\mathcal{S}_{G}^{(0) I, I I}+\mathcal{S}_{g f}=\frac{1}{2} \sum_{\mu \nu} \int_{-\pi}^{\pi} \frac{d^{4} k}{(2 \pi)^{4}}\left(\bar{A}_{\mu}(k) M_{\mu \nu}(k) \bar{A}_{\nu}(-k)\right),
$$

where

$$
\begin{aligned}
M_{00} & =\chi\left[\frac{\chi^{2}}{\alpha_{g}} \hat{k}_{0}^{2}+\sum_{j} \hat{k}_{j}^{2} q_{0 j}\right] \\
M_{j j} & =\frac{1}{\chi}\left[\frac{1}{\alpha_{g}} \hat{k}_{j}^{2}+\chi^{2} \hat{k}_{0}^{2} q_{0 j}+\sum_{l \neq j} \hat{k}_{l}^{2} q_{l j}\right] \\
M_{i \neq j} & =\frac{1}{\chi}\left[\frac{1}{\alpha_{g}} \hat{k}_{i} \hat{k}_{j}-\hat{k}_{i} \hat{k}_{j} q_{i j}\right]
\end{aligned}
$$




$$
M_{0 j}=M_{j 0}=\chi\left[\frac{1}{\alpha_{g}} \hat{k}_{0} \hat{k}_{j}-\hat{k}_{0} \hat{k}_{j} q_{0 j}\right]
$$

and

$$
\hat{k}_{\mu} \equiv 2 \sin \left(\frac{k_{\mu}}{2}\right)
$$

The $q_{\mu \nu}$ need to be specified only for $\mu \neq \nu$ and one has

$$
\begin{array}{rlrl}
q_{\mu \nu} & =1-c_{1}^{G}\left(\hat{k}_{\mu}^{2}+\hat{k}_{\nu}^{2}\right) & \mu \neq \nu & \mathcal{S}_{G}^{I} \\
q_{i j}=1+\frac{1}{12}\left(\hat{k}_{i}^{2}+\hat{k}_{j}^{2}\right) & i \neq j & \mathcal{S}_{G}^{I I} \\
q_{0 j}=1+\frac{1}{12} \hat{k}_{j}^{2} & \mathcal{S}_{G}^{I I}
\end{array}
$$

We have inverted the $4 \times 4$ matrix $M_{\mu \nu}$ using Mathematica keeping $q_{\mu \nu}$ general. For both gauge actions, $\mathcal{S}_{G}^{I}$ and $\mathcal{S}_{G}^{I I}$ the free gauge propagator has the structure

$$
D_{\mu \nu}(k)=M_{\mu \nu}^{-1}=\frac{1}{\left(\hat{k}^{2}\right)^{2}}\left[\alpha_{g} \hat{k}_{\mu} \hat{k}_{\nu} \chi+\frac{f_{N}^{\mu \nu}\left(\hat{k}_{\rho}, q_{\rho \sigma}, \chi\right)}{f_{D}\left(\hat{k}_{\rho}, q_{\rho \sigma}, \chi\right)}\right],
$$

with

$$
\hat{k}^{2}=\chi^{2} \hat{k}_{0}^{2}+\sum_{j} \hat{k}_{j}^{2}
$$

The term proportional to the gauge fixing parameter $\alpha_{g}$ has the familiar form

$$
\alpha_{g} \frac{\hat{k}_{\mu} \hat{k}_{\nu}}{\left(\hat{k}^{2}\right)^{2}} \chi=\alpha_{g} \frac{a_{\mu} a_{\nu}}{a_{s}^{3} a_{t}} \frac{\hat{k}_{\mu} \hat{k}_{\nu} /\left(a_{\mu} a_{\nu}\right)}{\left[\left(\hat{k}_{0} / a_{t}\right)^{2}+\sum_{j}\left(\hat{k}_{j} / a_{s}\right)^{2}\right]^{2}}
$$

with the conversion factor $a_{\mu} a_{\nu} / a_{s}^{3} a_{t}$ mentioned in (57). This factor results because we are looking at the propagator for dimensionless gauge fields $a_{\mu} A_{\mu}$ and because we carried out a dimensionless Fourier transform. The second term in (A17) is much more complicated. If one writes

$$
\begin{aligned}
f_{N}^{00}\left(\hat{k}_{\rho}, q_{\rho \sigma}, \chi\right) & =\frac{1}{\chi} \tilde{f}_{N}^{00} \\
f_{N}^{j j}\left(\hat{k}_{\rho}, q_{\rho \sigma}, \chi\right) & =\chi \tilde{f}_{N}^{j j} \\
f_{N}^{i \neq j}\left(\hat{k}_{\rho}, q_{\rho \sigma}, \chi\right) & =\chi \hat{k}_{i} \hat{k}_{j} \tilde{f}_{N}^{i \neq j} \\
f_{N}^{0 j}\left(\hat{k}_{\rho}, q_{\rho \sigma}, \chi\right) & =\chi \hat{k}_{0} \hat{k}_{j} \tilde{f}_{N}^{0 j}
\end{aligned}
$$

one can show that $f_{D}$ and all the $\tilde{f}_{N}^{\mu \nu}$ are functions only of $\left(\chi \hat{k}_{0}\right)^{2}, \hat{k}_{j}^{2}, q_{\rho \sigma}$ with no other $\chi$ dependence or odd powers of $\hat{k}_{\rho}$. We have not shown color indices in the above expressions. The gluon propagator is diagonal in color. 
Quark Propagator

The inverse free quark propagator for $\mathcal{S}_{D 234}^{I}$ is given by

$$
a_{t} \bar{G}_{0}^{-1}(k)=i \gamma_{0} K_{0}\left(k_{0}\right) \sin \left(k_{0}\right)+i \frac{C_{0}}{\chi} \sum_{j} \gamma_{j} K_{j}\left(k_{j}\right) \sin \left(k_{j}\right)+\Omega\left(k_{0}, \vec{k}\right)
$$

with

$$
\begin{aligned}
& K_{0}=1+\frac{C_{3 t}}{3}-\frac{C_{3 t}}{3} \cos \left(k_{0}\right) \\
& K_{j}=1+\frac{C_{3}}{3}-\frac{C_{3}}{3} \cos \left(k_{j}\right)
\end{aligned}
$$

and

$$
\begin{aligned}
\Omega= & \chi\left[2\left(1+\frac{C_{4 t}}{3}\right) \sin ^{2}\left(\frac{k_{0}}{2}\right)-\frac{C_{4 t}}{6} \sin ^{2}\left(k_{0}\right)\right] \\
& \frac{1}{\chi} \sum_{j}\left[2\left(1+\frac{C_{4}}{3}\right) \sin ^{2}\left(\frac{k_{j}}{2}\right)-\frac{C_{4}}{6} \sin ^{2}\left(k_{j}\right)\right]+a_{t} m .
\end{aligned}
$$

Propagators for the other quark actions can be obtained by setting the appropriate $C_{i(t)}$ equal to zero. Quark propagators are diagonal in color.

\section{Vertex Functions}

In deriving the one- and two-gluon emission vertices we have used the method described in [22]. We list again results only for $\mathcal{S}_{D 234}^{I}$. Those for other quark actions follow trivially. The general form for a single gluon emission vertex is

$$
V_{\mu}\left(k^{\prime}, k\right) \equiv-i \gamma_{\mu} W_{\mu}-W_{\mu}^{\prime}-\sum_{\nu} \sigma_{\nu \mu} W_{\nu \mu}^{\prime \prime}
$$

where $\mu$ is the polarization of the emitted gluon, $k^{\prime}$ the momentum of the outgoing quark and $k$ the momentum of the incoming quark. We suppress the color factor $T_{b c}^{a}$ which should multiply each of the above terms. Using the variables

$$
k_{\mu}^{ \pm} \equiv \frac{1}{2}\left(k^{\prime} \pm k\right)_{\mu}
$$

one has

$$
\begin{aligned}
& W_{0}=\left(1+\frac{C_{3 t}}{3}\right) \cos \left(k_{0}^{+}\right)-\frac{C_{3 t}}{3} \cos \left(2 k_{0}^{+}\right) \cos \left(k_{0}^{-}\right) \\
& W_{j}=\frac{C_{0}}{\chi}\left[\left(1+\frac{C_{3}}{3}\right) \cos \left(k_{j}^{+}\right)-\frac{C_{3}}{3} \cos \left(2 k_{j}^{+}\right) \cos \left(k_{j}^{-}\right)\right] \\
& W_{0}^{\prime}=\chi\left[\left(1+\frac{C_{4 t}}{3}\right) \sin \left(k_{0}^{+}\right)-\frac{C_{4 t}}{6} \sin \left(2 k_{0}^{+}\right) \cos \left(k_{0}^{-}\right)\right] \\
& W_{j}^{\prime}=\frac{1}{\chi}\left[\left(1+\frac{C_{4}}{3}\right) \sin \left(k_{j}^{+}\right)-\frac{C_{4}}{6} \sin \left(2 k_{j}^{+}\right) \cos \left(k_{j}^{-}\right)\right]
\end{aligned}
$$


and

$$
\begin{aligned}
W_{j 0}^{\prime \prime} & =\frac{1}{2} \sin \left(2 k_{j}^{-}\right) \cos \left(k_{0}^{-}\right) \frac{1}{3}\left[5-\cos \left(2 k_{j}^{-}\right)-\cos \left(2 k_{0}^{-}\right)\right] \\
W_{0 j}^{\prime \prime} & =\frac{1}{2} \sin \left(2 k_{0}^{-}\right) \cos \left(k_{j}^{-}\right) \frac{1}{3}\left[5-\cos \left(2 k_{j}^{-}\right)-\cos \left(2 k_{0}^{-}\right)\right] \\
W_{i j}^{\prime \prime} & =\frac{1}{2} \frac{1}{\chi} \sin \left(2 k_{i}^{-}\right) \cos \left(k_{j}^{-}\right) \frac{1}{3}\left[5-\cos \left(2 k_{i}^{-}\right)-\cos \left(2 k_{j}^{-}\right)\right] .
\end{aligned}
$$

For the clover action the factor $\frac{1}{3}\left[5-\cos \left(2 k_{\mu}^{-}\right)-\cos \left(2 k_{\nu}^{-}\right)\right]$in $W_{\mu \nu}^{\prime \prime}$ should be replaced by 1 . For the two-gluon emission vertex we do not present the most general result, but restrict ourselves to those terms necessary for the tadpole diagram $\Sigma^{\text {tad }}$. For instance, the $\sigma_{\mu \nu} F_{\mu \nu}$ term does not contribute to the tadpole diagram. We also omit terms that vanish upon symmetrizing between the two gluons. If $V_{\mu_{1} \mu_{2}}^{(2)}\left(k^{\prime}, k, q_{1}, q_{2}\right)$ stands for the emission vertex for gluons of momentum $q_{i}$ and polarization $\mu_{i}$, with $k_{\mu}=k_{\mu}^{\prime}+q_{1, \mu}+q_{2, \mu}$, one has

$$
\begin{aligned}
V_{00}^{(2)}= & \frac{i}{2} \gamma_{0}\left[\left(1+\frac{C_{3 t}}{3}\right) \sin \left(k_{0}^{+}\right)-\frac{2}{3} C_{3 t} \sin \left(2 k_{0}^{+}\right) \cos \left(\frac{q_{1,0}}{2}\right) \cos \left(\frac{q_{2,0}}{2}\right)\right] \\
& -\frac{\chi}{2}\left[\left(1+\frac{C_{4 t}}{3}\right) \cos \left(k_{0}^{+}\right)-\frac{1}{3} C_{4 t} \cos \left(2 k_{0}^{+}\right) \cos \left(\frac{q_{1,0}}{2}\right) \cos \left(\frac{q_{2,0}}{2}\right)\right] \\
V_{j j}^{(2)}= & \frac{i}{2} \frac{C_{0}}{\chi} \gamma_{j}\left[\left(1+\frac{C_{3}}{3}\right) \sin \left(k_{j}^{+}\right)-\frac{2}{3} C_{3} \sin \left(2 k_{j}^{+}\right) \cos \left(\frac{q_{1, j}}{2}\right) \cos \left(\frac{q_{2, j}}{2}\right)\right] \\
& -\frac{1}{2 \chi}\left[\left(1+\frac{C_{4}}{3}\right) \cos \left(k_{j}^{+}\right)-\frac{1}{3} C_{4} \cos \left(2 k_{j}^{+}\right) \cos \left(\frac{q_{1, j}}{2}\right) \cos \left(\frac{q_{2, j}}{2}\right)\right] .
\end{aligned}
$$

The color factor for these vertex functions is $\left(T^{a_{1}} T^{a_{2}}\right)_{b c}$.

\section{APPENDIX B: IR SUBTRACTIONS}

In this Appendix we list the IR subtraction, $\mathcal{F}_{\text {sub }}$ of equation (61), necessary to control numerical integration of IR divergent integrals. A gluon mass, $\lambda / a_{s}$, is introduced into $D_{\mu \nu}$ by replacing the first factor in (A17) by

$$
\frac{1}{\left(\hat{k}^{2}\right)^{2}} \rightarrow \frac{1}{\hat{k}^{2}} \frac{1}{\hat{k}^{2}+\lambda^{2}} .
$$

The lattice wave function renormalization $Z_{2}$ must reproduce the same IR divergence structure as in continuum QCD. For $Z_{2}^{-1}$ at one-loop the IR divergence is

$$
\begin{array}{ll}
\frac{\alpha_{s}}{3 \pi}\left[-1-\left(\alpha_{g}-1\right)\right] \ln \left(\lambda^{2}\right) & m=0 \\
\frac{\alpha_{s}}{3 \pi}\left[2-\left(\alpha_{g}-1\right)\right] \ln \left(\lambda^{2}\right) & m>0
\end{array}
$$

We note that by the $m=0$ theory we mean one in which the quark mass has been set to zero before taking the limit $\lambda \rightarrow 0$. This is the usual practice in much of the literature on 
massless lattice perturbation theory. Alternatively one could take the limit $a m \rightarrow 0$ and $\lambda \rightarrow 0$ keeping $a m \geq \lambda$. Since we want to compare with some of the massless literature with improved glue actions (e.g. [12]) we adopt the first definition in this article. In our massive calculations we do not go to extremely small masses and have not attempted to isolate $\ln (a m)$ contributions.

For our VEGAS integrations it was convenient to separate the $d / d p_{0}$ derivative in (61) into two parts

$$
\frac{d}{d p_{0}}\left[V_{\mu} \frac{\bar{G}_{0}}{a_{t}} V_{\nu}\right] \equiv \frac{d}{d p_{0}}\left[\frac{V G V_{\text {num }}}{V G V_{\text {den }}}\right]=\frac{V G V_{\text {num }}^{\prime}}{V G V_{\text {den }}}-\frac{V G V_{\text {num }}}{\left(V G V_{\text {den }}\right)^{2}} V G V_{d e n}^{\prime}
$$

Corresponding to the two parts with derivatives acting on the numerator or denominator, respectively, we introduce two separate subtraction terms $\mathcal{F}_{\text {sub }}^{\text {num }}$ and $\mathcal{F}_{\text {sub }}^{\text {den }}$. These are obtained by calculating the self energy diagram in continuum Euclidean perturbation theory with an appropriate mass $m_{\text {eff }}$ and the mass-shell condition $p=\left(i m_{e f f}, \overrightarrow{0}\right)$. The effective mass follows from expanding the lattice integrand in (61) about small $k$ and comparing with the continuum calculation [13]. It is given in (64). After converting to the dimensionless integration variables $k_{\mu}$ of $(\sqrt{61})$ one has for the part with the derivative acting on the denominator

$$
\begin{aligned}
& \mathcal{F}_{\text {sub }}^{\text {den }}=\theta\left(\Lambda^{2}-k^{2}\right) \\
& \times\left\{\frac{-4 \chi\left(\chi^{2} k_{0}^{2}+b^{2} / 4\right)\left(\left(k^{2}\right)^{2}-b^{2} \chi^{2} k_{0}^{2}\right)}{\left(k^{2}+\lambda^{2}\right)\left(\left(k^{2}\right)^{2}+b^{2} \chi^{2} k_{0}^{2}\right)^{2}}+\left(\alpha_{g}-1\right) \chi \frac{\chi^{2} k_{0}^{2}\left(b^{2}+2 k^{2}\right)}{k^{2}\left(k^{2}+\lambda^{2}\right)\left(\left(k^{2}\right)^{2}+b^{2} \chi^{2} k_{0}^{2}\right)}\right\},
\end{aligned}
$$

with

$$
k^{2}=\chi^{2} k_{0}^{2}+\sum_{j} k_{j}^{2} \quad b=2 a_{s} m_{\text {eff }} .
$$

The $\theta$-function imposes a cutoff on $\mathcal{F}_{\text {sub }}^{\text {den }}$ so that it vanishes identically for $k^{2}>\Lambda^{2}$, where $\Lambda$ is some number $0<\Lambda \leq \pi$. The subtraction term can be integrated analytically to give

$$
\begin{aligned}
& F^{\text {den }}\left(m_{\text {eff }}>0, \Lambda, \lambda\right)=\int_{k} \mathcal{F}_{\text {sub }}^{\text {den }}\left(k, m_{\text {eff }}>0, \Lambda, \lambda\right) \\
& =\frac{\alpha_{s}}{3 \pi}\left\{\left[-2 \ln \left(\frac{\Lambda^{2}}{\lambda^{2}}\right)+2 \ln \left(\frac{\Lambda+\sqrt{b^{2}+\Lambda^{2}}}{b}\right)+\frac{4 \Lambda^{2}}{b^{4}}\left(b^{2}+3 \Lambda^{2}\right)+\frac{\sqrt{b^{2}+\Lambda^{2}}}{b^{4}} 2 \Lambda\left(b^{2}-6 \Lambda^{2}\right)\right]\right. \\
& \left.+\left(\alpha_{g}-1\right)\left[\ln \left(\frac{\Lambda^{2}}{\lambda^{2}}\right)+\frac{2 \Lambda^{2}}{b^{4}}\left(\Lambda^{2}+2 b^{2}\right)-\frac{\Lambda\left(2 \Lambda^{2}+3 b^{2}\right)}{b^{4}} \sqrt{b^{2}+\Lambda^{2}}-\ln \left(\frac{\Lambda+\sqrt{b^{2}+\Lambda^{2}}}{b}\right)\right]\right\}
\end{aligned}
$$

and

$$
F^{d e n}\left(m_{e f f} \equiv 0, \Lambda, \lambda\right)=\frac{\alpha_{s}}{3 \pi}\left[-1+\frac{\alpha_{g}-1}{2}\right] \ln \left(\frac{\Lambda^{2}}{\lambda^{2}}\right) .
$$

The contribution in (B3) from the derivative acting on the numerator is 


$$
\mathcal{F}_{\text {sub }}^{\text {num }}=\theta\left(\Lambda^{2}-k^{2}\right)\left\{\frac{2 \chi k^{2}}{\left(\left(k^{2}\right)^{2}+b^{2} \chi^{2} k_{0}^{2}\right)\left(k^{2}+\lambda^{2}\right)}+\left(\alpha_{g}-1\right) \chi \frac{k^{2}-2 \chi^{2} k_{0}^{2}}{\left(\left(k^{2}\right)^{2}+b^{2} \chi^{2} k_{0}^{2}\right)\left(k^{2}+\lambda^{2}\right)}\right\}
$$

which leads to

$$
\begin{aligned}
& F^{\text {num }}\left(m_{\text {eff }}>0, \Lambda, \lambda\right)=\int_{k} \mathcal{F}_{\text {sub }}^{\text {num }}\left(k, m_{\text {eff }}>0, \Lambda, \lambda\right) \\
& =\frac{\alpha_{s}}{3 \pi}\left\{\left[4 \ln \left(\frac{\Lambda+\sqrt{b^{2}+\Lambda^{2}}}{b}\right)-\frac{4 \Lambda^{2}}{b^{2}}+\frac{4 \Lambda}{b^{2}} \sqrt{b^{2}+\Lambda^{2}}\right]\right. \\
& \left.\quad+\left(\alpha_{g}-1\right)\left[\ln \left(\frac{\Lambda+\sqrt{b^{2}+\Lambda^{2}}}{b}\right)-\frac{2 \Lambda^{2}}{b^{4}}\left(\Lambda^{2}+2 b^{2}\right)+\frac{\Lambda\left(2 \Lambda^{2}+3 b^{2}\right)}{b^{4}} \sqrt{b^{2}+\Lambda^{2}}\right]\right\}
\end{aligned}
$$

and

$$
F^{\text {num }}\left(m_{\text {eff }} \equiv 0, \Lambda, \lambda\right)=\frac{\alpha_{s}}{3 \pi}\left[2+\frac{\alpha_{g}-1}{2}\right] \ln \left(\frac{\Lambda^{2}}{\lambda^{2}}\right) .
$$

$F^{d e n}+F^{\text {num }}$ reproduces the IR divergent logarithms of (B2). 


\section{REFERENCES}

[1] C. Morningstar and M. Peardon; Phys. Rev. D60:034509 (1999).

[2] T. Manke et al.; Phys. Rev. Lett 82:4396 (1999).

[3] I. Drummond et al.; hep-lat/9912041.

[4] CP-PACS Collaboration, T. Manke et al.; hep-lat/9909038.

[5] B. Mertens, A. Kronfeld and A. El-Khadra; Phys. Rev. D58: 034505 (1998).

[6] M. Alford, T. Klassen and G. P. Lepage; Phys. Rev. D58:034503 (1998), Nucl. Phys. B496 377 (1997).

[7] G.P. Lepage; Nucl. Phys. B (Proc. Suppl.)60A 267 (1998).

[8] G.P. Lepage and P.B. Mackenzie, Phys. Rev. D48, 2250 (1993).

[9] P. Weisz; Nucl. Phys. B212 1 (1983); P. Weisz and R. Wohlert; Nucl. Phys. B236 397 (1984) ; erratum, ibid. B247 544 (1984).

[10] Y. Iwasaki, preprint, UTHEP-118 (Dec. 1983), unpublished.

[11] B. Sheikholeslami and R. Wohlert, Nucl. Phys. B259, 572 (1985).

[12] S. Aoki et al.; Phys. Rev. D58:074505 (1998).

[13] Y. Kuramashi; Phys. Rev. D58:034507 (1998).

[14] M. Lüscher; Comm. Math. Phys. 54283 (1977).

[15] A. El-Khadra, A. Kronfeld and P. Mackenzie; Phys. Rev. D55 3933 (1997).

[16] G.P. Lepage, J. Comput. Phys. 27, 192 (1978).

[17] E. Eichten and B. Hill; Phys. Lett. B240, 193 (1990).

[18] M. Alford, T. Klassen and G.P. Lepage; Nucl. Phys. B(Proc.Suppl)53, 861 (1997)

[19] M. Alford, R. Horgan et al.; in preparation.

[20] P. Bouchard, C. Lin and O. Pene; Phys. Rev. D40, 1529 (1989); D41, 3541(E) (1990).

[21] A. Borrelli and C. Pittori; Nucl. Phys. B385, 502 (1992).

[22] C. Morningstar; Phys. Rev. D48, 2265 (1993). 


\section{TABLES}

\begin{tabular}{ccc}
\hline \hline \multicolumn{1}{c}{ action } & comments & parameters \\
\hline $\mathcal{S}^{A}=\mathcal{S}_{G}^{I}+\mathcal{S}_{\text {clover }}$ & massive and massless & $c_{0}^{G}=5 / 3, c_{1}^{G}=-1 / 12, \chi=1$ \\
$\mathcal{S}^{A^{\prime}}=\mathcal{S}_{G}^{I}+\mathcal{S}_{\text {clover }}$ & massive and massless & $c_{0}^{G}=3.648, c_{1}^{G}=-0.331, \chi=1$ \\
$\mathcal{S}^{B}=\mathcal{S}_{G}^{I I}+\mathcal{S}_{\text {clover }}$ & massive and massless & $\chi \geq 1$ \\
$\mathcal{S}^{C}=\mathcal{S}_{G}^{I}+\mathcal{S}_{D 234}^{I}$ & massless & $c_{0}^{G}=5 / 3, c_{1}^{G}=-1 / 12, \chi=1$ \\
$\mathcal{S}^{D}=\mathcal{S}_{G}^{I I}+\mathcal{S}_{D 234}^{I I}$ & massive and massless & $\chi \geq 1$ \\
\hline \hline
\end{tabular}

TABLE I. Combinations of gauge and quark actions considered in this article. 


\begin{tabular}{c|rcrrrr}
\hline \hline \multicolumn{7}{c}{ Action $\mathcal{S}^{A}$} \\
\hline & regular & tadpole & reg + tad & t.i. & $a_{s} M_{1, \text { nosub }}^{(1)}$ & $a_{s} M_{1, \text { sub }}^{(1)}$ \\
\hline$a_{s} M_{1}^{(0)}$ & \multicolumn{7}{c}{$\alpha_{g}=1.0$} \\
\hline 0.00 & $-1.770(3)$ & 4.298 & $2.528(3)$ & -3.001 & $-0.472(3)$ & 0.000 \\
0.01 & $-1.718(5)$ & 4.266 & $2.548(5)$ & -2.978 & $-0.430(5)$ & $0.037(6)$ \\
0.05 & $-1.547(3)$ & 4.141 & $2.594(3)$ & -2.891 & $-0.297(3)$ & $0.152(4)$ \\
0.10 & $-1.369(3)$ & 3.991 & $2.623(3)$ & -2.786 & $-0.164(3)$ & $0.263(4)$ \\
0.50 & $-0.463(3)$ & 3.030 & $2.567(3)$ & -2.115 & $0.451(3)$ & $0.737(3)$ \\
1.00 & $0.092(3)$ & 2.260 & $2.352(3)$ & -1.578 & $0.774(3)$ & $0.948(3)$ \\
2.00 & $0.530(3)$ & 1.511 & $2.041(3)$ & -1.055 & $0.986(3)$ & $1.050(3)$ \\
5.00 & $0.743(3)$ & 1.096 & $1.839(3)$ & -0.765 & $1.074(3)$ & $1.077(3)$ \\
10.00 & $0.752(3)$ & 1.075 & $1.827(3)$ & -0.750 & $1.077(3)$ & $1.077(3)$ \\
\hline$a_{s} M_{1}^{(0)}$ & & $\alpha_{g}=0.0$ & & & \\
\hline 0.00 & $-0.472(3)$ & 3.001 & $2.528(3)$ & -3.001 & $-0.472(3)$ & 0.000 \\
0.01 & $-0.429(5)$ & 2.978 & $2.549(5)$ & -2.978 & $-0.429(5)$ & $0.038(6)$ \\
0.05 & $-0.293(3)$ & 2.891 & $2.597(3)$ & -2.891 & $-0.293(3)$ & $0.156(4)$ \\
0.10 & $-0.161(3)$ & 2.786 & $2.626(3)$ & -2.786 & $-0.161(3)$ & $0.266(4)$ \\
0.50 & $0.454(3)$ & 2.115 & $2.569(3)$ & -2.115 & $0.454(3)$ & $0.740(3)$ \\
1.00 & $0.775(3)$ & 1.578 & $2.353(3)$ & -1.578 & $0.775(3)$ & $0.949(3)$ \\
2.00 & $0.987(3)$ & 1.055 & $2.042(3)$ & -1.055 & $0.987(3)$ & $1.051(3)$ \\
5.00 & $1.076(3)$ & 0.765 & $1.841(3)$ & -0.765 & $1.076(3)$ & $1.079(3)$ \\
10.00 & $1.079(3)$ & 0.750 & $1.829(3)$ & -0.750 & $1.079(3)$ & $1.079(3)$ \\
\hline \hline
\end{tabular}

TABLE II. One-loop mass renormalization for action $\mathcal{S}^{A}$ for different $a_{s} M_{1}^{(0)}$ values. Results are presented for two choices of the gauge parameter $\alpha_{g}=1$ and $\alpha_{g}=0 . a_{s} M_{1, \text { nosub }}^{(1)}$ is the same as $\left(\mathrm{reg}+\operatorname{tad}+\right.$ t.i.). $a_{s} M_{1, \text { sub }}^{(1)}$ is defined in (40) and related to $a_{s} M_{1, \text { nosub }}^{(1)}$ in (78). Where errors are not indicated explicitly, they are of $O(1)$ or less in the last digit. 


\begin{tabular}{c|ccc|ccc}
\hline \hline & \multicolumn{3}{|c|}{ Action $\mathcal{S}^{A^{\prime}}$} & \multicolumn{3}{c}{ Action $\mathcal{S}^{C}$} \\
\hline$a_{s} M_{1}^{(0)}$ & reg $+\operatorname{tad}$ & $a_{s} M_{1, \text { nosub }}^{(1)}$ & $a_{s} M_{1, \text { sub }}^{(1)}$ & $\operatorname{reg}+\operatorname{tad}$ & $a_{s} M_{1, \text { nosub }}^{(1)}$ & $a_{s} M_{1, \text { sub }}^{(1)}$ \\
\hline 0.00 & 1.480 & -0.397 & 0.000 & 2.213 & -1.287 & 0.000 \\
0.01 & 1.505 & -0.358 & 0.035 & & \\
0.05 & 1.566 & -0.242 & 0.136 & & \\
0.10 & 1.612 & -0.131 & 0.228 & & \\
0.50 & 1.690 & 0.367 & 0.608 & & \\
1.00 & 1.611 & 0.624 & 0.770 & & \\
2.00 & 1.466 & 0.806 & 0.859 & & \\
5.00 & 1.376 & 0.897 & 0.900 & & \\
10.00 & 1.371 & 0.901 & 0.901 & & \\
\hline \hline
\end{tabular}

TABLE III. One-loop mass renormalization for actions $\mathcal{S}^{A^{\prime}}$ and $\mathcal{S}^{C}$. Numerical integration errors are at the \pm 0.006 level for $a_{s} M_{1}^{(0)}=0.01$ and of $O(4)$ or less in the last digit otherwise. 


\begin{tabular}{|c|c|c|c|c|c|c|}
\hline \multicolumn{7}{|c|}{ Action $\mathcal{S}^{B}$} \\
\hline & reg + tad & $a_{s} M_{1, \text { nosub }}^{(1)}$ & $a_{s} M_{1, s u b}^{(1)}$ & reg + tad & $a_{s} M_{1, \text { nosub }}^{(1)}$ & $a_{s} M_{1, s u b}^{(1)}$ \\
\hline$\overline{a_{s} M_{1}^{(0)}}$ & & $\chi=1.0$ & & & $\chi=2.0$ & \\
\hline 0.00 & 2.710 & -0.479 & 0.000 & 3.240 & -0.881 & 0.000 \\
\hline 0.01 & 2.729 & -0.436 & 0.038 & 3.250 & -0.833 & 0.039 \\
\hline 0.05 & 2.774 & -0.299 & 0.157 & 3.260 & -0.675 & 0.164 \\
\hline 0.10 & 2.799 & -0.164 & 0.269 & 3.251 & -0.512 & 0.288 \\
\hline 0.50 & 2.717 & 0.463 & 0.754 & 3.060 & 0.290 & 0.863 \\
\hline 1.00 & 2.466 & 0.779 & 0.955 & 2.824 & 0.772 & 1.178 \\
\hline 2.00 & 2.096 & 0.961 & 1.026 & 2.461 & 1.155 & 1.381 \\
\hline 5.00 & 1.842 & 1.014 & 1.017 & 1.978 & 1.371 & 1.419 \\
\hline$a_{s} M_{1}^{(0)}$ & & $\chi=3.0$ & & & $\chi=3.6$ & \\
\hline 0.00 & 3.298 & -1.139 & 0.000 & 3.286 & -1.243 & 0.000 \\
\hline 0.01 & 3.302 & -1.092 & 0.036 & 3.289 & -1.195 & 0.036 \\
\hline 0.05 & 3.312 & -0.918 & 0.167 & 3.299 & -1.017 & 0.167 \\
\hline 0.10 & 3.299 & -0.742 & 0.293 & 3.287 & -0.835 & 0.295 \\
\hline 0.50 & 3.110 & 0.145 & 0.896 & 3.106 & 0.083 & 0.905 \\
\hline 1.00 & 2.901 & 0.695 & 1.244 & 2.910 & 0.656 & 1.262 \\
\hline 2.00 & 2,589 & 1.161 & 1.498 & 2.620 & 1.151 & 1.532 \\
\hline 5.00 & 2.106 & 1.472 & 1.582 & 2.158 & 1.496 & 1.636 \\
\hline$\overline{a_{s} M_{1}^{(0)}}$ & & $\chi=4.0$ & & & $\chi=5.0$ & \\
\hline 0.00 & 3.271 & -1.299 & 0.000 & 3.233 & -1.405 & 0.000 \\
\hline 0.01 & 3.271 & -1.255 & 0.031 & 3.233 & -1.360 & 0.031 \\
\hline 0.05 & 3.285 & -1.070 & 0.167 & 3.249 & -1.170 & 0.168 \\
\hline 0.10 & 3.274 & -0.885 & 0.296 & 3.239 & -0.980 & 0.297 \\
\hline 0.50 & 3.099 & 0.049 & 0.910 & 3.076 & -0.018 & 0.915 \\
\hline 1.00 & 2.911 & 0.634 & 1.270 & 2.903 & 0.590 & 1.283 \\
\hline 2.00 & 2.631 & 1.143 & 1.547 & 2.645 & 1.124 & 1.572 \\
\hline 5.00 & 2.184 & 1.505 & 1.662 & 2.228 & 1.518 & 1.707 \\
\hline$\overline{a_{s} M_{1}^{(0)}}$ & & $\chi=5.3$ & & & $\chi=6.0$ & \\
\hline 0.00 & 3.222 & -1.429 & 0.000 & 3.197 & -1.479 & 0.000 \\
\hline 0.01 & 3.226 & -1.380 & 0.035 & 3.204 & -1.426 & 0.038 \\
\hline 0.05 & 3.238 & -1.194 & 0.167 & 3.213 & -1.241 & 0.168 \\
\hline 0.10 & 3.228 & -1.003 & 0.296 & 3.205 & -1.048 & 0.296 \\
\hline 0.50 & 3.069 & -0.034 & 0.915 & 3.053 & -0.065 & 0.918 \\
\hline 1.00 & 2.899 & 0.579 & 1.285 & 2.891 & 0.557 & 1.290 \\
\hline 2.00 & 2.647 & 1.119 & 1.577 & 2.649 & 1.108 & 1.586 \\
\hline 5.00 & 2.237 & 1.520 & 1.717 & 2.254 & 1.522 & 1.734 \\
\hline
\end{tabular}

TABLE IV. One-loop mass renormalization for action $\mathcal{S}^{B}$ for several values of the anisotropy $\chi=a_{s} / a_{t}$. Numerical integration errors are as in Table III. 


\begin{tabular}{|c|c|c|c|c|c|c|}
\hline \multicolumn{7}{|c|}{ Action $\mathcal{S}^{D}$} \\
\hline & reg + tad & $a_{s} M_{1, \text { nosub }}^{(1)}$ & $a_{s} M_{1, s u b}^{(1)}$ & reg + tad & $a_{s} M_{1, \text { nosub }}^{(1)}$ & $a_{s} M_{1, s u b}^{(1)}$ \\
\hline$a_{s} M_{1}^{(0)}$ & & $\chi=1.0$ & & & $\chi=2.0$ & \\
\hline 0.00 & 2.247 & -1.339 & 0.000 & 2.740 & -1.927 & 0.000 \\
\hline 0.01 & 2.268 & -1.289 & 0.037 & 2.744 & -1.878 & 0.030 \\
\hline 0.05 & 2.329 & -1.121 & 0.153 & 2.776 & -1.678 & 0.157 \\
\hline 0.10 & 2.375 & -0.947 & 0.265 & 2.785 & -1.473 & 0.277 \\
\hline 0.50 & 2.451 & -0.043 & 0.769 & 2.728 & -0.396 & 0.858 \\
\hline 1.00 & 2.342 & 0.510 & 1.003 & 2.623 & 0.320 & 1.208 \\
\hline 2.00 & 2.082 & 0.894 & 1.075 & 2.404 & 0.958 & 1.453 \\
\hline 5.00 & 1.843 & 1.012 & 1.021 & 1.986 & 1.349 & 1.455 \\
\hline$\overline{a_{s} M_{1}^{(0)}}$ & & $\chi=3.0$ & & & $\chi=3.6$ & \\
\hline 0.00 & 2.788 & -2.250 & 0.000 & 2,777 & -2.370 & 0.000 \\
\hline 0.01 & 2.794 & -2.195 & 0.033 & 2.781 & -2.315 & 0.032 \\
\hline 0.05 & 2.818 & -1.984 & 0.159 & 2.805 & -2.099 & 0.158 \\
\hline 0.10 & 2.824 & -1.762 & 0.282 & 2.811 & -1.872 & 0.282 \\
\hline 0.50 & 2.759 & -0.601 & 0.883 & 2.751 & -0.680 & 0.888 \\
\hline 1.00 & 2.674 & 0.178 & 1.262 & 2.675 & 0.120 & 1.275 \\
\hline 2.00 & 2.506 & 0.901 & 1.566 & 2.528 & 0.870 & 1.596 \\
\hline 5.00 & 2.115 & 1.423 & 1.639 & 2.166 & 1.435 & 1.701 \\
\hline$\overline{a_{s} M_{1}^{(0)}}$ & & $\chi=4.0$ & & & $\chi=5.0$ & \\
\hline 0.00 & 2.769 & -2.427 & 0.000 & 2.732 & -2.544 & 0.000 \\
\hline 0.01 & 2.768 & -2.377 & 0.026 & 2.734 & -2.490 & 0.029 \\
\hline 0.05 & 2.792 & -2.152 & 0.159 & 2.760 & -2.267 & 0.156 \\
\hline 0.10 & 2.799 & -1.928 & 0.278 & 2.768 & -2.030 & 0.282 \\
\hline 0.50 & 2.742 & -0.722 & 0.886 & 2.719 & -0.798 & 0.891 \\
\hline 1.00 & 2.672 & 0.090 & 1.279 & 2.660 & 0.033 & 1.288 \\
\hline 2.00 & 2.536 & 0.853 & 1.609 & 2.542 & 0.817 & 1.629 \\
\hline 5.00 & 2.190 & 1.436 & 1.729 & 2.232 & 1.436 & 1.779 \\
\hline$\overline{a_{s} M_{1}^{(0)}}$ & & $\chi=5.3$ & & & $\chi=6.0$ & \\
\hline 0.00 & 2.720 & -2.572 & 0.000 & 2.701 & -2.620 & 0.000 \\
\hline 0.01 & 2.724 & -2.516 & 0.031 & 2.708 & -2.561 & 0.033 \\
\hline 0.05 & 2.759 & -2.283 & 0.166 & 2.732 & -2.337 & 0.158 \\
\hline 0.10 & 2.765 & -2.047 & 0.291 & 2.740 & -2.099 & 0.283 \\
\hline 0.50 & 2.717 & -0.810 & 0.899 & 2.699 & -0.848 & 0.894 \\
\hline 1.00 & 2.658 & 0.022 & 1.293 & 2.646 & -0.007 & 1.291 \\
\hline 2.00 & 2.543 & 0.809 & 1.633 & 2.541 & 0.791 & 1.638 \\
\hline 5.00 & 2.242 & 1.436 & 1.791 & 2.256 & 1.432 & 1.808 \\
\hline
\end{tabular}

TABLE V. One-loop mass renormalization for action $\mathcal{S}^{D}$ for several values of the anisotropy $\chi=a_{s} / a_{t}$. Numerical integration errors are as in Table III. 


\begin{tabular}{|c|c|c|c|c|c|c|}
\hline \multicolumn{7}{|c|}{ Action $\mathcal{S}^{A}$} \\
\hline & \multicolumn{3}{|c|}{$\alpha_{g}=1.0$} & \multicolumn{3}{|c|}{$\alpha_{g}=0.0$} \\
\hline$a_{s} M_{1}^{(0)}$ & $Z_{2, d p_{0}}^{(1)}$ no $t . i$ & $Z_{2, d p_{0}}^{(1)}$ with t.i. & $Z_{2}^{(1)}$ with $t . i$ & $Z_{2, d p_{0}}^{(1)}$ no $t . i$. & $Z_{2, d p_{0}}^{(1)}$ with $t . i$. & $Z_{2}^{(1)}$ with t.i. \\
\hline 0.00 & $0.687(3)$ & $-0.063(3)$ & $-0.063(3)$ & $1.195(3)$ & $0.445(3)$ & $0.445(3)$ \\
\hline 0.01 & $-2.688(6)$ & $-3.438(6)$ & $-3.475(7)$ & $-2.169(6)$ & $-2.919(6)$ & $-2.956(7)$ \\
\hline 0.05 & $-1.569(5)$ & $-2.319(5)$ & $-2.471(6)$ & $-1.052(5)$ & $-1.802(5)$ & $-1.954(6)$ \\
\hline 0.10 & $-1.041(5)$ & $-1.791(5)$ & $-2.054(6)$ & $-0.524(5)$ & $-1.274(5)$ & $-1.537(6)$ \\
\hline 0.50 & $0.393(3)$ & $-0.357(3)$ & $-1.094(4)$ & $0.905(3)$ & $0.155(3)$ & $-0.582(4)$ \\
\hline 1.00 & $1.112(3)$ & $0.362(3)$ & $-0.586(4)$ & $1.623(3)$ & $0.873(3)$ & $-0.075(4)$ \\
\hline 2.00 & $1.795(3)$ & $1.045(3)$ & $-0.005(4)$ & $2.304(3)$ & $1.554(3)$ & $0.504(4)$ \\
\hline 5.00 & $2.223(20)$ & $1.473(20)$ & $0.396(20)$ & $2.719(20)$ & $1.969(20)$ & $0.892(20)$ \\
\hline
\end{tabular}

TABLE VI. One-loop wave function renormalization for action $\mathcal{S}^{A}$ for $\operatorname{different} a_{s} M_{1}^{(0)}$ values. Results are presented for two choices for the gauge parameter $\alpha_{g}=1$ and $\alpha_{g}=0 . Z_{2}^{(1)}$ includes both $Z_{2, d p_{0}}^{(1)}$ and $Z_{2, M_{1}}^{(1)}$ as defined in equation (82).

\begin{tabular}{|c|c|c|}
\hline & Action $\mathcal{S}^{A}$ & \\
\hline$\overline{a_{s} M_{1}^{(0)}}$ & $Z_{2, d i f f}^{(1)}$ no $t . i$. & $Z_{2, d i f f}^{(1)}$ with $t . i$. \\
\hline 0.00 & $0.634(3)$ & $-0.116(3)$ \\
\hline 0.01 & $0.668(6)$ & $-0.082(6)$ \\
\hline 0.05 & $0.763(5)$ & $0.013(5)$ \\
\hline 0.10 & $0.849(5)$ & $0.099(5)$ \\
\hline 0.50 & $1.259(3)$ & $0.509(3)$ \\
\hline 1.00 & $1.536(3)$ & $0.786(3)$ \\
\hline 2.00 & $1.778(3)$ & $1.028(3)$ \\
\hline 5.00 & $1.623(20)$ & $0.873(20)$ \\
\hline
\end{tabular}

TABLE VII. $Z_{2, d p_{0}}^{(1)}$ with $\ln (a m)$ contributions subtracted out for action $\mathcal{S}^{A}$ in Feynman gauge. $Z_{2, \text { diff }}^{(1)}$ is defined in equation (84). 


\begin{tabular}{|c|c|c|c|c|c|c|}
\hline \multicolumn{7}{|c|}{ Action $\mathcal{S}^{A^{\prime}}$} \\
\hline & \multicolumn{3}{|c|}{$\alpha_{g}=1.0$} & \multicolumn{3}{|c|}{$\alpha_{g}=0.0$} \\
\hline$a_{s} M_{1}^{(0)}$ & $Z_{2, d p_{0}}^{(1)}$ no $t . i$ & $Z_{2, d p_{0}}^{(1)}$ with $t . i$. & $Z_{2}^{(1)}$ with $t . i$ & $Z_{2, d p_{0}}^{(1)}$ no $t . i$. & $Z_{2, d p_{0}}^{(1)}$ with $t . i$. & $Z_{2}^{(1)}$ with $t . i$. \\
\hline 0.00 & 0.239 & -0.230 & -0.230 & 0.747 & 0.278 & 0.278 \\
\hline 0.01 & -3.137 & -3.606 & -3.641 & -2.628 & -3.097 & -3.132 \\
\hline 0.05 & -2.024 & -2.493 & -2.629 & -1.515 & -1.984 & -2.120 \\
\hline 0.10 & -1.502 & -1.971 & -2.199 & -0.993 & -1.462 & -1.690 \\
\hline 0.50 & -0.120 & -0.590 & -1.198 & 0.389 & -0.081 & -0.689 \\
\hline 1.00 & 0.546 & 0.076 & -0.694 & 1.054 & 0.585 & -0.185 \\
\hline 2.00 & 1.165 & 0.695 & -0.164 & 1.674 & 1.204 & 0.345 \\
\hline 5.00 & 1.568 & 1.099 & 0.199 & 2.079 & 1.610 & 0.710 \\
\hline \multicolumn{7}{|c|}{ Action $\mathcal{S}^{C}$} \\
\hline & \multicolumn{3}{|c|}{$\alpha_{g}=1.0$} & \multicolumn{3}{|c|}{$\alpha_{g}=0.0$} \\
\hline$a_{s} M_{1}^{(0)}$ & $Z_{2, d p_{0}}^{(1)}$ no t.i. & $Z_{2, d p_{0}}^{(1)}$ with $t . i$. & $Z_{2}^{(1)}$ with $t . i$ & $Z_{2, d p_{0}}^{(1)}$ no t.i. & $Z_{2, d p_{0}}^{(1)}$ with $t . i$. & $Z_{2}^{(1)}$ with $t . i$. \\
\hline 0.00 & 0.145 & -0.355 & -0.355 & 0.653 & 0.153 & 0.153 \\
\hline
\end{tabular}

TABLE VIII. One-loop wave function renormalization for actions $\mathcal{S}^{A^{\prime}}$ and $\mathcal{S}^{C}$. Numerical integration errors are at the \pm 0.02 level for $a_{s} M_{1}^{(0)}=5.0$, at the \pm 0.006 level for $a_{s} M_{1}^{(0)}=0.01$, 0.05 and 0.10 and of $O(4)$ in the last digit for other masses. 


\begin{tabular}{|c|c|c|c|c|c|c|}
\hline \multicolumn{7}{|c|}{ Action $\mathcal{S}^{B} \quad \alpha_{g}=1.0$} \\
\hline & $Z_{2, d p_{0}}^{(1)}$ no t.i. & $Z_{2, d p_{0}}^{(1)}$ with $t . i$. & $Z_{2}^{(1)}$ with t.i. & $Z_{2, d p_{0}}^{(1)}$ no t.i. & $Z_{2, d p_{0}}^{(1)}$ with $t . i$. & $Z_{2}^{(1)}$ with t.i. \\
\hline$\overline{a_{s} M_{1}^{(0)}}$ & \multicolumn{3}{|c|}{$\chi=1.0$} & \multicolumn{3}{|c|}{$\chi=2.0$} \\
\hline 0.00 & 0.789 & -0.023 & -0.023 & 0.323 & 0.110 & 0.110 \\
\hline 0.01 & -2.586 & -3.398 & -3.431 & -3.050 & -3.263 & -3.293 \\
\hline 0.05 & -1.469 & -2.281 & -2.437 & -1.930 & -2.143 & -2.301 \\
\hline 0.10 & -0.942 & -1.755 & -2.024 & -1.406 & -1.619 & -1.887 \\
\hline 0.50 & 0.467 & -0.345 & -1.099 & -0.111 & -0.324 & -0.974 \\
\hline 1.00 & 1.150 & 0.337 & -0.618 & 0.398 & 0.185 & -0.569 \\
\hline 2.00 & 1.766 & 0.953 & -0.073 & 0.822 & 0.609 & -0.147 \\
\hline 5.00 & 2.124 & 1.312 & 0.295 & 1.237 & 1.025 & 0.312 \\
\hline$\overline{a_{s} M_{1}^{(0)}}$ & \multicolumn{3}{|c|}{$\chi=3.0$} & \multicolumn{3}{|c|}{$\chi=3.6$} \\
\hline 0.00 & 0.265 & 0.172 & 0.172 & 0.253 & 0.190 & 0.190 \\
\hline 0.01 & -3.107 & -3.200 & -3.231 & -3.118 & -3.182 & -3.213 \\
\hline 0.05 & -1.988 & -2.080 & -2.240 & -1.999 & -2.062 & -2.222 \\
\hline 0.10 & -1.465 & -1.558 & -1.828 & -1.476 & -1.540 & -1.810 \\
\hline 0.50 & -0.198 & -0.290 & -0.923 & -0.216 & -0.279 & -0.907 \\
\hline 1.00 & 0.259 & 0.167 & -0.534 & 0.228 & 0.165 & -0.522 \\
\hline 2.00 & 0.594 & 0.501 & -0.150 & 0.536 & 0.473 & -0.147 \\
\hline 5.00 & 0.911 & 0.819 & 0.272 & 0.807 & 0.744 & 0.256 \\
\hline$a_{s} M_{1}^{(0)}$ & \multicolumn{3}{|c|}{$\chi=4.0$} & \multicolumn{3}{|c|}{$\chi=5.0$} \\
\hline 0.00 & 0.249 & 0.198 & 0.198 & 0.242 & 0.210 & 0.210 \\
\hline 0.01 & -3.123 & -3.174 & -3.205 & -3.130 & -3.162 & -3.193 \\
\hline 0.05 & -2.003 & -2.054 & -2.214 & -2.010 & -2.042 & -2.202 \\
\hline 0.10 & -1.481 & -1.532 & -1.803 & -1.487 & -1.519 & -1.790 \\
\hline 0.50 & -0.223 & -0.275 & -0.901 & -0.233 & -0.266 & -0.889 \\
\hline 1.00 & 0.215 & 0.164 & -0.517 & 0.196 & 0.164 & -0.508 \\
\hline 2.00 & 0.511 & 0.460 & -0.146 & 0.473 & 0.441 & -0.143 \\
\hline 5.00 & 0.758 & 0.707 & 0.249 & 0.678 & 0.645 & 0.236 \\
\hline$a_{s} M_{1}^{(0)}$ & \multicolumn{3}{|c|}{$\chi=5.3$} & \multicolumn{3}{|c|}{$\chi=6.0$} \\
\hline 0.00 & 0.241 & 0.212 & 0.212 & 0.238 & 0.216 & 0.216 \\
\hline 0.01 & -3.131 & -3.160 & -3.191 & -3.133 & -3.156 & -3.186 \\
\hline 0.05 & -2.011 & -2.040 & -2.200 & -2.013 & -2.036 & -2.196 \\
\hline 0.10 & -1.488 & -1.517 & -1.788 & -1.490 & -1.513 & -1.783 \\
\hline 0.50 & -0.236 & -0.264 & -0.887 & -0.239 & -0.261 & -0.882 \\
\hline 1.00 & 0.193 & 0.164 & -0.506 & 0.186 & 0.164 & -0.502 \\
\hline 2.00 & 0.466 & 0.437 & -0.142 & 0.453 & 0.431 & -0.140 \\
\hline 5.00 & 0.661 & 0.633 & 0.234 & 0.631 & 0.609 & 0.229 \\
\hline
\end{tabular}

TABLE IX. One-loop wave function renormalization for action $\mathcal{S}^{D}$ in Feynman gauge. Numerical integration errors are at the \pm 0.02 level for $a_{s} M_{1}^{(0)}=5.0$ and $\chi<3$, at the \pm 0.006 level for $a_{s} M_{1}^{(0)}=0.01,0.05$ and 0.10 and of $O(4)$ in the last digit for all other cases. 


\begin{tabular}{|c|c|c|c|c|c|c|}
\hline \multicolumn{7}{|c|}{ Action $\mathcal{S}^{B} \quad \alpha_{g}=0.0$} \\
\hline & $Z_{2, d p_{0}}^{(1)}$ no t.i. & $Z_{2, d p_{0}}^{(1)}$ with $t . i$. & $Z_{2}^{(1)}$ with $t . i$ & $Z_{2, d p_{0}}^{(1)}$ no $t . i$ & $Z_{2, d p_{0}}^{(1)}$ with $t . i$ & $Z_{2}^{(1)}$ with $t . i$. \\
\hline$\overline{a_{s} M_{1}^{(0)}}$ & \multicolumn{3}{|c|}{$\chi=1.0$} & \multicolumn{3}{|c|}{$\chi=2.0$} \\
\hline 0.00 & 1.297 & 0.485 & 0.485 & 0.833 & 0.620 & 0.620 \\
\hline 0.01 & -2.077 & -2.889 & -2.930 & -2.539 & -2.751 & -2.793 \\
\hline 0.05 & -0.960 & -1.772 & -1.931 & -1.419 & -1.632 & -1.794 \\
\hline 0.10 & -0.433 & -1.246 & -1.518 & -0.895 & -1.108 & -1.379 \\
\hline 0.50 & 0.975 & 0.163 & -0.593 & 0.400 & 0.187 & -0.465 \\
\hline 1.00 & 1.658 & 0.845 & -0.111 & 0.908 & 0.696 & -0.058 \\
\hline 2.00 & 2.274 & 1.461 & 0.434 & 1.332 & 1.119 & 0.363 \\
\hline 5.00 & 2.635 & 1.823 & 0.804 & 1.748 & 1.535 & 0.822 \\
\hline$a_{s} M_{1}^{(0)}$ & \multicolumn{3}{|c|}{$\chi=3.0$} & \multicolumn{3}{|c|}{$\chi=3.6$} \\
\hline 0.00 & 0.763 & 0.670 & 0.670 & 0.747 & 0.683 & 0.683 \\
\hline 0.01 & -2.608 & -2.701 & -2.742 & -2.624 & -2.688 & -2.728 \\
\hline 0.05 & -1.489 & -1.581 & -1.745 & -1.505 & -1.568 & -1.732 \\
\hline 0.10 & -0.966 & -1.059 & -1.332 & -0.982 & -1.045 & -1.319 \\
\hline 0.50 & 0.301 & 0.208 & -0.426 & 0.279 & 0.215 & -0.415 \\
\hline 1.00 & 0.757 & 0.665 & -0.037 & 0.722 & 0.658 & -0.029 \\
\hline 2.00 & 1.092 & 0.999 & 0.348 & 1.029 & 0.966 & 0.346 \\
\hline 5.00 & 1.408 & 1.316 & 0.770 & 1.301 & 1.237 & 0.750 \\
\hline$\overline{a_{s} M_{1}^{(0)}}$ & \multicolumn{3}{|c|}{$\chi=4.0$} & \multicolumn{3}{|c|}{$\chi=5.0$} \\
\hline 0.00 & 0.740 & 0.689 & 0.689 & 0.729 & 0.697 & 0.697 \\
\hline 0.01 & -2.631 & -2.682 & -2.722 & -2.642 & -2.674 & -2.713 \\
\hline 0.05 & -1.511 & -1.562 & -1.726 & -1.522 & -1.554 & -1.718 \\
\hline 0.10 & -0.988 & -1.040 & -1.313 & -0.999 & -1.031 & -1.305 \\
\hline 0.50 & 0.269 & 0.217 & -0.410 & 0.255 & 0.222 & -0.403 \\
\hline 1.00 & 0.706 & 0.655 & -0.026 & 0.684 & 0.651 & -0.021 \\
\hline 2.00 & 1.002 & 0.951 & 0.345 & 0.960 & 0.928 & 0.345 \\
\hline 5.00 & 1.249 & 1.198 & 0.740 & 1.165 & 1.133 & 0.724 \\
\hline$a_{s} M_{1}^{(0)}$ & \multicolumn{3}{|c|}{$\chi=5.3$} & \multicolumn{3}{|c|}{$\chi=6.0$} \\
\hline 0.00 & 0.727 & 0.698 & 0.698 & 0.723 & 0.701 & 0.701 \\
\hline 0.01 & -2.644 & -2.673 & -2.712 & -2.648 & -2.670 & -2.709 \\
\hline 0.05 & -1.524 & -1.553 & -1.716 & -1.528 & -1.550 & -1.714 \\
\hline 0.10 & -1.001 & -1.030 & -1.303 & -1.005 & -1.027 & -1.301 \\
\hline 0.50 & 0.252 & 0.223 & -0.401 & 0.247 & 0.225 & -0.398 \\
\hline 1.00 & 0.679 & 0.650 & -0.020 & 0.672 & 0.649 & -0.017 \\
\hline 2.00 & 0.952 & 0.923 & 0.344 & 0.937 & 0.915 & 0.345 \\
\hline 5.00 & 1.148 & 1.119 & 0.720 & 1.116 & 1.093 & 0.714 \\
\hline
\end{tabular}

TABLE X. One-loop wave function renormalization for action $\mathcal{S}^{B}$ in Landau gauge. Numerical integration errors are as in Table IX. 


\begin{tabular}{|c|c|c|c|c|c|c|}
\hline \multicolumn{7}{|c|}{ Action $\mathcal{S}^{D} \quad \alpha_{g}=1.0$} \\
\hline & $Z_{2, d p_{0}}^{(1)}$ no $t . i$ & $Z_{2, d p_{0}}^{(1)}$ with $t . i$. & $Z_{2}^{(1)}$ with t.i. & $Z_{2, d p_{0}}^{(1)}$ no t.i. & $Z_{2, d p_{0}}^{(1)}$ with $t . i$. & $Z_{2}^{(1)}$ with t.i. \\
\hline$a_{s} M_{1}^{(0)}$ & \multicolumn{3}{|c|}{$\chi=1.0$} & \multicolumn{3}{|c|}{$\chi=2.0$} \\
\hline 0.00 & 0.619 & -0.194 & -0.194 & 0.102 & -0.111 & -0.111 \\
\hline 0.01 & -2.755 & -3.568 & -3.597 & -3.270 & -3.482 & -3.511 \\
\hline 0.05 & -1.636 & -2.448 & -2.601 & -2.146 & -2.358 & -2.510 \\
\hline 0.10 & -1.107 & -1.920 & -2.185 & -1.617 & -1.830 & -2.088 \\
\hline 0.50 & 0.320 & -0.493 & -1.262 & -0.287 & -0.500 & -1.146 \\
\hline 1.00 & 1.034 & 0.221 & -0.781 & 0.258 & 0.045 & -0.728 \\
\hline 2.00 & 1.710 & 0.898 & -0.178 & 0.735 & 0.523 & -0.272 \\
\hline 5.00 & 2.138 & 1.325 & 0.304 & 1.217 & 1.005 & 0.274 \\
\hline$\overline{a_{s} M_{1}^{(0)}}$ & \multicolumn{3}{|c|}{$\chi=3.0$} & \multicolumn{3}{|c|}{$\chi=3.6$} \\
\hline 0.00 & 0.038 & -0.054 & -0.054 & 0.027 & -0.036 & -0.036 \\
\hline 0.01 & -3.332 & -3.425 & -3.454 & -3.344 & -3.407 & -3.436 \\
\hline 0.05 & -2.209 & -2.302 & -2.452 & -2.221 & -2.284 & -2.435 \\
\hline 0.10 & -1.682 & -1.775 & -2.033 & -1.694 & -1.757 & -2.015 \\
\hline 0.50 & -0.383 & -0.476 & -1.098 & -0.403 & -0.467 & -1.082 \\
\hline 1.00 & 0.112 & 0.019 & -0.692 & 0.078 & 0.015 & -0.679 \\
\hline 2.00 & 0.499 & 0.406 & -0.274 & 0.438 & 0.375 & -0.271 \\
\hline 5.00 & 0.878 & 0.786 & 0.220 & 0.770 & 0.707 & 0.200 \\
\hline$a_{s} M_{1}^{(0)}$ & \multicolumn{3}{|c|}{$\chi=4.0$} & \multicolumn{3}{|c|}{$\chi=5.0$} \\
\hline 0.00 & 0.023 & -0.028 & -0.028 & 0.016 & -0.016 & -0.016 \\
\hline 0.01 & -3.348 & -3.399 & -3.428 & -3.354 & -3.387 & -3.415 \\
\hline 0.05 & -2.225 & -2.276 & -2.426 & -2.231 & -2.264 & -2.414 \\
\hline 0.10 & -1.698 & -1.749 & -2.007 & -1.705 & -1.737 & -1.995 \\
\hline 0.50 & -0.412 & -0.463 & -1.075 & -0.424 & -0.456 & -1.063 \\
\hline 1.00 & 0.064 & 0.013 & -0.674 & 0.043 & 0.011 & -0.663 \\
\hline 2.00 & 0.412 & 0.361 & -0.269 & 0.372 & 0.340 & -0.265 \\
\hline 5.00 & 0.720 & 0.668 & 0.191 & 0.635 & 0.603 & 0.176 \\
\hline$a_{s} M_{1}^{(0)}$ & \multicolumn{3}{|c|}{$\chi=5.3$} & \multicolumn{3}{|c|}{$\chi=6.0$} \\
\hline 0.00 & 0.015 & -0.014 & -0.014 & 0.013 & -0.009 & -0.009 \\
\hline 0.01 & -3.355 & -3.384 & -3.413 & -3.358 & -3.380 & -3.409 \\
\hline 0.05 & -2.233 & -2.261 & -2.411 & -2.235 & -2.257 & -2.407 \\
\hline 0.10 & -1.706 & -1.735 & -1.992 & -1.709 & -1.731 & -1.988 \\
\hline 0.50 & -0.426 & -0.455 & -1.061 & -0.430 & -0.452 & -1.057 \\
\hline 1.00 & 0.039 & 0.010 & -0.661 & 0.031 & 0.009 & -0.658 \\
\hline 2.00 & 0.364 & 0.335 & -0.264 & 0.350 & 0.328 & -0.262 \\
\hline 5.00 & 0.618 & 0.589 & 0.173 & 0.586 & 0.563 & 0.168 \\
\hline
\end{tabular}

TABLE XI. One-loop wave function renormalization for action $\mathcal{S}^{D}$ in Feynman gauge. Numerical integration errors are as in Table IX. 


\begin{tabular}{|c|c|c|c|c|c|c|}
\hline \multicolumn{7}{|c|}{ Action $\mathcal{S}^{D} \quad \alpha_{g}=0.0$} \\
\hline & $Z_{2, d p_{0}}^{(1)}$ no $t . i$. & $Z_{2, d p_{0}}^{(1)}$ with $t . i$. & $Z_{2}^{(1)}$ with t.i. & $Z_{2, d p_{0}}^{(1)}$ no t.i. & $Z_{2, d p_{0}}^{(1)}$ with $t . i$. & $Z_{2}^{(1)}$ with t.i. \\
\hline$a_{s} M_{1}^{(0)}$ & \multicolumn{3}{|c|}{$\chi=1.0$} & \multicolumn{3}{|c|}{$\chi=2.0$} \\
\hline 0.00 & 1.127 & 0.314 & 0.314 & 0.612 & 0.399 & 0.399 \\
\hline 0.01 & -2.246 & -3.058 & -3.097 & -2.758 & -2.971 & -3.007 \\
\hline 0.05 & -1.127 & -1.939 & -2.095 & -1.635 & -1.847 & -2.003 \\
\hline 0.10 & -0.598 & -1.410 & -1.679 & -1.106 & -1.319 & -1.580 \\
\hline 0.50 & 0.828 & 0.016 & -0.756 & 0.223 & 0.011 & -0.637 \\
\hline 1.00 & 1.542 & 0.730 & -0.274 & 0.768 & 0.555 & -0.219 \\
\hline 2.00 & 2.219 & 1.407 & 0.330 & 1.246 & 1.033 & 0.238 \\
\hline 5.00 & 2.646 & 1.834 & 0.811 & 1.727 & 1.514 & 0.784 \\
\hline$a_{s} M_{1}^{(0)}$ & \multicolumn{3}{|c|}{$\chi=3.0$} & \multicolumn{3}{|c|}{$\chi=3.6$} \\
\hline 0.00 & 0.537 & 0.444 & 0.444 & 0.521 & 0.457 & 0.457 \\
\hline 0.01 & -2.833 & -2.926 & -2.960 & -2.849 & -2.913 & -2.947 \\
\hline 0.05 & -1.710 & -1.803 & -1.959 & -1.727 & -1.790 & -1.946 \\
\hline 0.10 & -1.183 & -1.276 & -1.537 & -1.200 & -1.263 & -1.524 \\
\hline 0.50 & 0.116 & 0.023 & -0.600 & 0.091 & 0.027 & -0.590 \\
\hline 1.00 & 0.610 & 0.518 & -0.194 & 0.572 & 0.509 & -0.186 \\
\hline 2.00 & 0.996 & 0.904 & 0.224 & 0.932 & 0.868 & 0.222 \\
\hline 5.00 & 1.376 & 1.283 & 0.717 & 1.263 & 1.200 & 0.693 \\
\hline$a_{s} M_{1}^{(0)}$ & \multicolumn{3}{|c|}{$\chi=4.0$} & \multicolumn{3}{|c|}{$\chi=5.0$} \\
\hline 0.00 & 0.514 & 0.463 & 0.463 & 0.504 & 0.471 & 0.471 \\
\hline 0.01 & -2.856 & -2.907 & -2.941 & -2.867 & -2.899 & -2.932 \\
\hline 0.05 & -1.733 & -1.785 & -1.940 & -1.744 & -1.776 & -1.932 \\
\hline 0.10 & -1.207 & -1.258 & -1.519 & -1.217 & -1.250 & -1.510 \\
\hline 0.50 & 0.080 & 0.029 & -0.585 & 0.064 & 0.031 & -0.578 \\
\hline 1.00 & 0.556 & 0.505 & -0.182 & 0.531 & 0.499 & -0.176 \\
\hline 2.00 & 0.903 & 0.852 & 0.222 & 0.859 & 0.827 & 0.223 \\
\hline 5.00 & 1.210 & 1.159 & 0.682 & 1.122 & 1.090 & 0.663 \\
\hline$a_{s} M_{1}^{(0)}$ & \multicolumn{3}{|c|}{$\chi=5.3$} & \multicolumn{3}{|c|}{$\chi=6.0$} \\
\hline 0.00 & 0.502 & 0.473 & 0.473 & 0.498 & 0.476 & 0.476 \\
\hline 0.01 & -2.868 & -2.897 & -2.929 & -2.872 & -2.895 & -2.926 \\
\hline 0.05 & -1.746 & -1.775 & -1.930 & -1.749 & -1.772 & -1.927 \\
\hline 0.10 & -1.219 & -1.248 & -1.509 & -1.223 & -1.246 & -1.506 \\
\hline 0.50 & 0.061 & 0.032 & -0.576 & 0.055 & 0.033 & -0.573 \\
\hline 1.00 & 0.526 & 0.497 & -0.175 & 0.517 & 0.495 & -0.173 \\
\hline 2.00 & 0.851 & 0.822 & 0.223 & 0.835 & 0.813 & 0.224 \\
\hline 5.00 & 1.104 & 1.075 & 0.660 & 1.071 & 1.049 & 0.654 \\
\hline
\end{tabular}

TABLE XII. One-loop wave function renormalization for action $\mathcal{S}^{D}$ in Landau gauge. Numerical integration errors are as in Table IX. 


\begin{tabular}{|c|c|c|c|c|}
\hline \multicolumn{5}{|c|}{ Action $\mathcal{S}^{B} \quad \chi=4.0$} \\
\hline & regular & tadpole & $C_{0}^{(1)}$ no t.i. $(\mathrm{reg}+\mathrm{tad})$ & $C_{0}^{(1)}$ with t.i. \\
\hline$\overline{a_{s} M_{1}^{(0)}}$ & \multicolumn{4}{|c|}{$\alpha_{g}=1.0$} \\
\hline 0.00 & $-0.618(3)$ & 1.474 & $0.856(3)$ & $-0.343(3)$ \\
\hline 0.01 & $-0.616(8)$ & 1.474 & $0.858(8)$ & $-0.341(8)$ \\
\hline 0.05 & $-0.617(5)$ & 1.474 & $0.857(5)$ & $-0.342(5)$ \\
\hline 0.10 & $-0.617(5)$ & 1.474 & $0.857(5)$ & $-0.342(5)$ \\
\hline 0.50 & $-0.608(3)$ & 1.474 & $0.866(3)$ & $-0.333(3)$ \\
\hline 1.00 & $-0.587(2)$ & 1.474 & $0.887(2)$ & $-0.312(2)$ \\
\hline 2.00 & $-0.547(2)$ & 1.474 & $0.927(2)$ & $-0.272(2)$ \\
\hline 5.00 & $-0.519(2)$ & 1.474 & $0.955(2)$ & $-0.244(2)$ \\
\hline 10.00 & $-0.561(2)$ & 1.474 & $0.913(2)$ & $-0.286(2)$ \\
\hline$a_{s} M_{1}^{(0)}$ & \multicolumn{4}{|c|}{$\alpha_{g}=0.0$} \\
\hline 0.00 & $-0.345(3)$ & 1.199 & $0.854(3)$ & $-0.345(3)$ \\
\hline 0.01 & $-0.351(8)$ & 1.199 & $0.848(8)$ & $-0.351(8)$ \\
\hline 0.05 & $-0.347(5)$ & 1.199 & $0.852(5)$ & $-0.347(5)$ \\
\hline 0.10 & $-0.347(5)$ & 1.199 & $0.852(5)$ & $-0.347(5)$ \\
\hline 0.50 & $-0.333(3)$ & 1.199 & $0.866(3)$ & $-0.333(3)$ \\
\hline 1.00 & $-0.312(2)$ & 1.199 & $0.887(2)$ & $-0.312(2)$ \\
\hline 2.00 & $-0.273(2)$ & 1.199 & $0.926(2)$ & $-0.273(2)$ \\
\hline 5.00 & $-0.244(2)$ & 1.199 & $0.955(2)$ & $-0.244(2)$ \\
\hline 10.00 & $-0.286(2)$ & 1.199 & $0.913(2)$ & $-0.286(2)$ \\
\hline
\end{tabular}

TABLE XIII. One-loop speed of light renormalization for action $\mathcal{S}^{B}$ for different $a_{s} M_{1}^{(0)}$ values at fixed anisotropy $\chi=4.0$. Where errors are not indicated explicitly, they are of $O(1)$ or less in the last digit. 


\begin{tabular}{|c|c|c|c|c|c|}
\hline \multicolumn{6}{|c|}{ Action $\mathcal{S}^{B}$} \\
\hline & $C_{0}^{(1)}$ no t.i. & $C_{0}^{(1)}$ with t.i. & $C_{0}^{(1)}$ no t.i. & $C_{0}^{(1)}$ & with t.i. \\
\hline$\overline{a_{s} M_{1}^{(0)}}$ & \multicolumn{2}{|c|}{$\chi=1.0$} & \multicolumn{3}{|c|}{$\chi=2.0$} \\
\hline 0.00 & -0.039 & -0.019 & 0.668 & & -0.210 \\
\hline 0.01 & -0.036 & -0.015 & 0.671 & & -0.207 \\
\hline 0.05 & -0.038 & -0.018 & 0.669 & & -0.208 \\
\hline 0.10 & -0.044 & -0.023 & 0.669 & & -0.209 \\
\hline 0.50 & -0.127 & -0.107 & 0.654 & & -0.223 \\
\hline 1.00 & -0.265 & -0.245 & 0.633 & & -0.244 \\
\hline 2.00 & -0.509 & -0.488 & 0.582 & & -0.295 \\
\hline 5.00 & -0.749 & -0.728 & 0.432 & & -0.446 \\
\hline$\overline{a_{s} M_{1}^{(0)}}$ & \multicolumn{2}{|c|}{$\chi=3.0$} & \multicolumn{3}{|c|}{$\chi=3.6$} \\
\hline 0.00 & 0.807 & -0.302 & 0.842 & & -0.330 \\
\hline 0.01 & 0.809 & -0.300 & 0.843 & & -0.328 \\
\hline 0.05 & 0.808 & -0.301 & 0.842 & & -0.329 \\
\hline 0.10 & 0.807 & -0.302 & 0.842 & & -0.330 \\
\hline 0.50 & 0.811 & -0.298 & 0.849 & & -0.322 \\
\hline 1.00 & 0.821 & -0.288 & 0.867 & & -0.304 \\
\hline 2.00 & 0.836 & -0.273 & 0.899 & & -0.272 \\
\hline 5.00 & 0.802 & -0.307 & 0.907 & & -0.264 \\
\hline$a_{s} M_{1}^{(0)}$ & \multicolumn{2}{|c|}{$\chi=4.0$} & \multicolumn{3}{|c|}{$\chi=5.0$} \\
\hline \begin{tabular}{l|}
0.00 \\
\end{tabular} & 0.856 & -0.343 & 0.880 & & -0.363 \\
\hline 0.01 & 0.858 & -0.341 & 0.882 & & -0.361 \\
\hline 0.05 & 0.857 & -0.342 & 0.881 & & -0.362 \\
\hline 0.10 & 0.857 & -0.342 & 0.881 & & -0.362 \\
\hline 0.50 & 0.866 & -0.333 & 0.893 & & -0.350 \\
\hline 1.00 & 0.887 & -0.312 & 0.918 & & -0.325 \\
\hline 2.00 & 0.927 & -0.272 & 0.969 & & -0.274 \\
\hline 5.00 & 0.955 & -0.244 & 1.031 & & -0.212 \\
\hline$a_{s} M_{1}^{(0)}$ & \multicolumn{2}{|c|}{$\chi=5.3$} & \multicolumn{3}{|c|}{$\chi=6.0$} \\
\hline \begin{tabular}{l|}
0.00 \\
\end{tabular} & 0.885 & -0.367 & 0.893 & & -0.374 \\
\hline 0.01 & 0.887 & -0.365 & 0.896 & & -0.372 \\
\hline 0.05 & 0.886 & -0.366 & 0.895 & & -0.373 \\
\hline 0.10 & 0.886 & -0.366 & 0.895 & & -0.373 \\
\hline 0.50 & 0.898 & -0.354 & 0.907 & & -0.360 \\
\hline 1.00 & 0.924 & -0.327 & 0.935 & & -0.332 \\
\hline 2.00 & 0.977 & -0.274 & 0.992 & & -0.275 \\
\hline 5.00 & 1.046 & -0.206 & 1.073 & & -0.194 \\
\hline
\end{tabular}

TABLE XIV. One-loop speed of light renormalization for action $\mathcal{S}^{B}$. Numerical integration errors are at the \pm 0.008 level for $a_{s} M_{1}^{(0)}=0.01$, at the \pm 0.005 level for $a_{s} M_{1}^{(0)}=0.05$ and 0.10 and at the \pm 0.003 level or less for other masses. 


\begin{tabular}{|c|c|c|c|c|}
\hline \multirow[b]{2}{*}{$a_{s} M_{1}^{(0)}$} & \multicolumn{2}{|c|}{ Action $\mathcal{S}^{A}$} & \multicolumn{2}{|c|}{ Action $\mathcal{S}^{A^{\prime}}$} \\
\hline & $C_{0}^{(1)}$ no t.i. & $C_{0}^{(1)}$ with t.i. & $C_{0}^{(1)}$ no t.i. & $C_{0}^{(1)}$ with t.i. \\
\hline 0.00 & 0.000 & 0.000 & 0.000 & 0.000 \\
\hline 0.01 & 0.000 & 0.000 & 0.000 & 0.000 \\
\hline 0.05 & 0.000 & 0.000 & 0.000 & 0.000 \\
\hline 0.10 & -0.007 & -0.007 & -0.007 & -0.007 \\
\hline 0.50 & -0.102 & -0.102 & -0.098 & -0.098 \\
\hline 1.00 & -0.258 & -0.258 & -0.243 & -0.243 \\
\hline 2.00 & -0.536 & -0.536 & -0.490 & -0.490 \\
\hline 5.00 & -0.809 & -0.809 & -0.730 & -0.730 \\
\hline
\end{tabular}

TABLE XV. One-loop speed of light renormalization for actions $\mathcal{S}^{A}$ and $\mathcal{S}^{A^{\prime}}$. Numerical integration errors are as in Table XIV. 


\begin{tabular}{|c|c|c|c|c|c|}
\hline \multicolumn{6}{|c|}{ Action $\mathcal{S}^{D}$} \\
\hline & $C_{0}^{(1)}$ no t.i. & $C_{0}^{(1)}$ with t.i. & $C_{0}^{(1)}$ no t.i. & $C_{0}^{(1)}$ & with t.i. \\
\hline$a_{s} M_{1}^{(0)}$ & \multicolumn{2}{|c|}{$\chi=1.0$} & \multicolumn{3}{|c|}{$\chi=2.0$} \\
\hline 0.00 & -0.424 & -0.139 & 0.213 & & -0.301 \\
\hline 0.01 & -0.421 & -0.137 & 0.216 & & -0.298 \\
\hline 0.05 & -0.424 & -0.139 & 0.213 & & -0.301 \\
\hline 0.10 & -0.430 & -0.146 & 0.211 & & -0.303 \\
\hline 0.50 & -0.525 & -0.241 & 0.179 & & -0.335 \\
\hline 1.00 & -0.683 & -0.398 & 0.129 & & -0.385 \\
\hline 2.00 & -0.971 & -0.686 & 0.033 & & -0.482 \\
\hline 5.00 & -1.275 & -0.991 & -0.180 & & -0.694 \\
\hline$a_{s} M_{1}^{(0)}$ & \multicolumn{2}{|c|}{$\chi=3.0$} & \multicolumn{3}{|c|}{$\chi=3.6$} \\
\hline 0.00 & 0.320 & -0.388 & 0.345 & & -0.415 \\
\hline 0.01 & 0.324 & -0.385 & 0.348 & & -0.412 \\
\hline 0.05 & 0.321 & -0.387 & 0.346 & & -0.414 \\
\hline 0.10 & 0.319 & -0.390 & 0.344 & & -0.415 \\
\hline 0.50 & 0.305 & -0.404 & 0.334 & & -0.426 \\
\hline 1.00 & 0.286 & -0.422 & 0.324 & & -0.436 \\
\hline 2.00 & 0.256 & -0.452 & 0.311 & & -0.448 \\
\hline 5.00 & 0.167 & -0.541 & 0.266 & & -0.494 \\
\hline$a_{s} M_{1}^{(0)}$ & \multicolumn{2}{|c|}{$\chi=4.0$} & \multicolumn{3}{|c|}{$\chi=5.0$} \\
\hline 0.00 & 0.356 & -0.427 & 0.372 & & -0.446 \\
\hline 0.01 & 0.358 & -0.425 & 0.375 & & -0.443 \\
\hline 0.05 & 0.356 & -0.426 & 0.373 & & -0.444 \\
\hline 0.10 & 0.355 & -0.427 & 0.372 & & -0.446 \\
\hline 0.50 & 0.347 & -0.436 & 0.367 & & -0.451 \\
\hline 1.00 & 0.340 & -0.443 & 0.365 & & -0.453 \\
\hline 2.00 & 0.335 & -0.447 & 0.372 & & -0.446 \\
\hline 5.00 & 0.311 & -0.471 & 0.382 & & -0.436 \\
\hline$a_{s} M_{1}^{(0)}$ & \multicolumn{2}{|c|}{$\chi=5.3$} & \multicolumn{3}{|c|}{$\chi=6.0$} \\
\hline 0.00 & 0.375 & -0.450 & 0.381 & & -0.457 \\
\hline 0.01 & 0.378 & -0.446 & 0.384 & & -0.453 \\
\hline 0.05 & 0.376 & -0.448 & 0.383 & & -0.455 \\
\hline 0.10 & 0.376 & -0.449 & 0.382 & & -0.455 \\
\hline 0.50 & 0.371 & -0.454 & 0.378 & & -0.459 \\
\hline 1.00 & 0.370 & -0.455 & 0.379 & & -0.459 \\
\hline 2.00 & 0.379 & -0.446 & 0.392 & & -0.446 \\
\hline 5.00 & 0.396 & -0.429 & 0.421 & & -0.416 \\
\hline
\end{tabular}

TABLE XVI. One-loop speed of light renormalization for action $\mathcal{S}^{D}$. Numerical integration errors are as in Table XIV. 


\section{FIGURES}

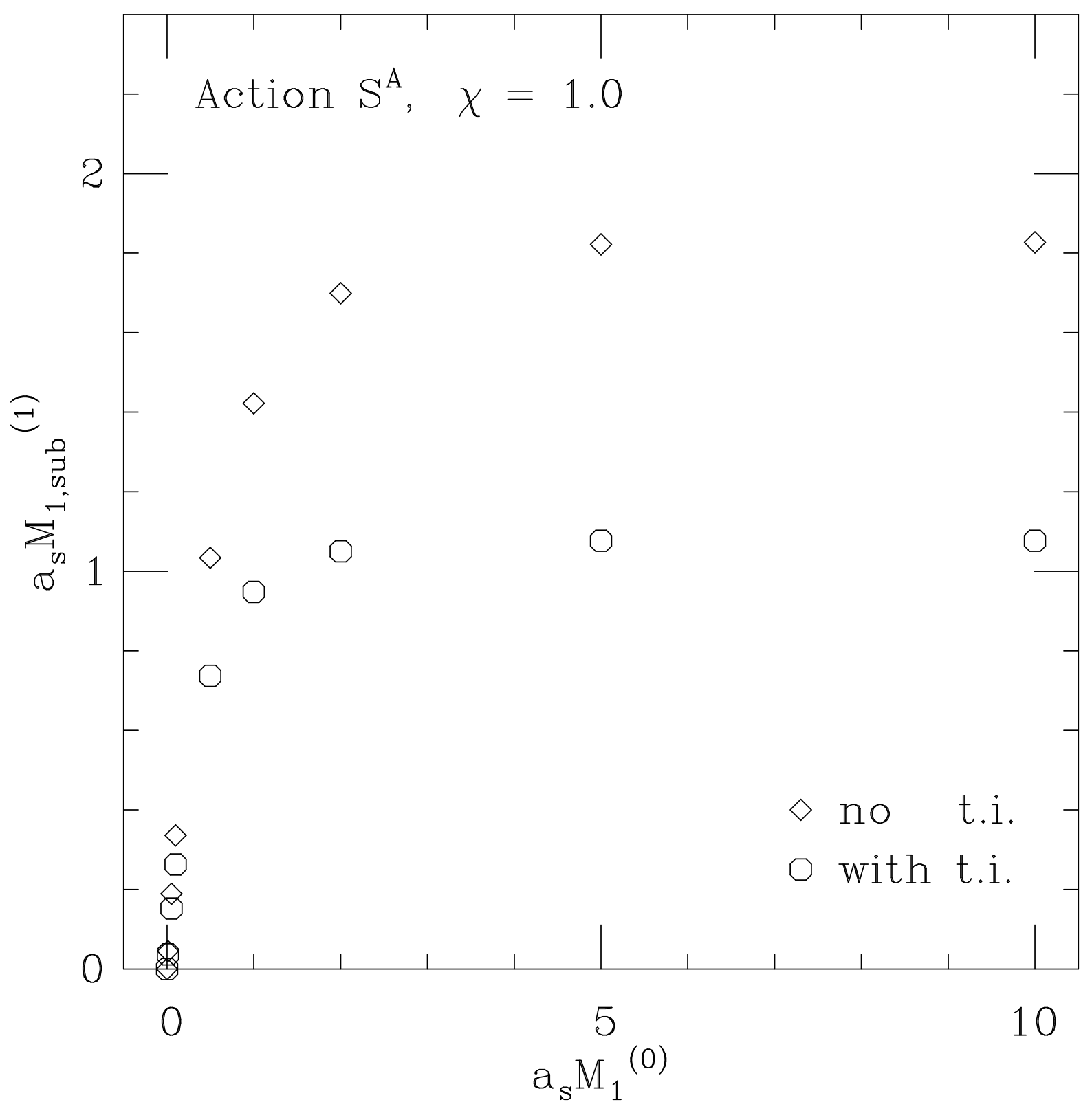

FIG. 1. $\quad a_{s} M_{1, s u b}^{(1)}$ versus $a_{s} M_{1}^{(0)}$ both with and without tadpole improvement for action $\mathcal{S}^{A}$. 


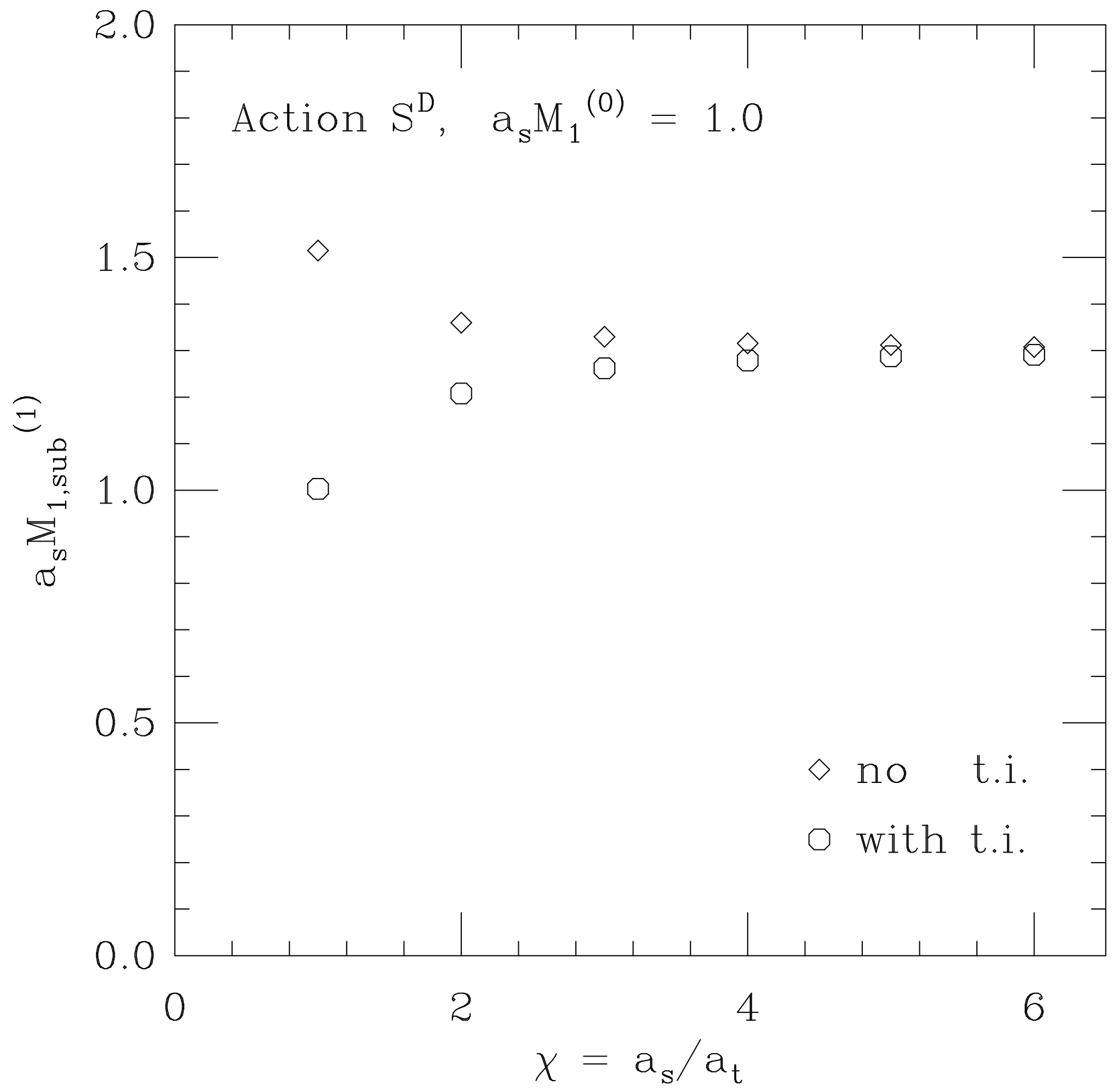

FIG. 2. $\quad a_{s} M_{1, \text { sub }}^{(1)}$ versus $\chi$ both with and without tadpole improvement for action $\mathcal{S}^{A}$. 


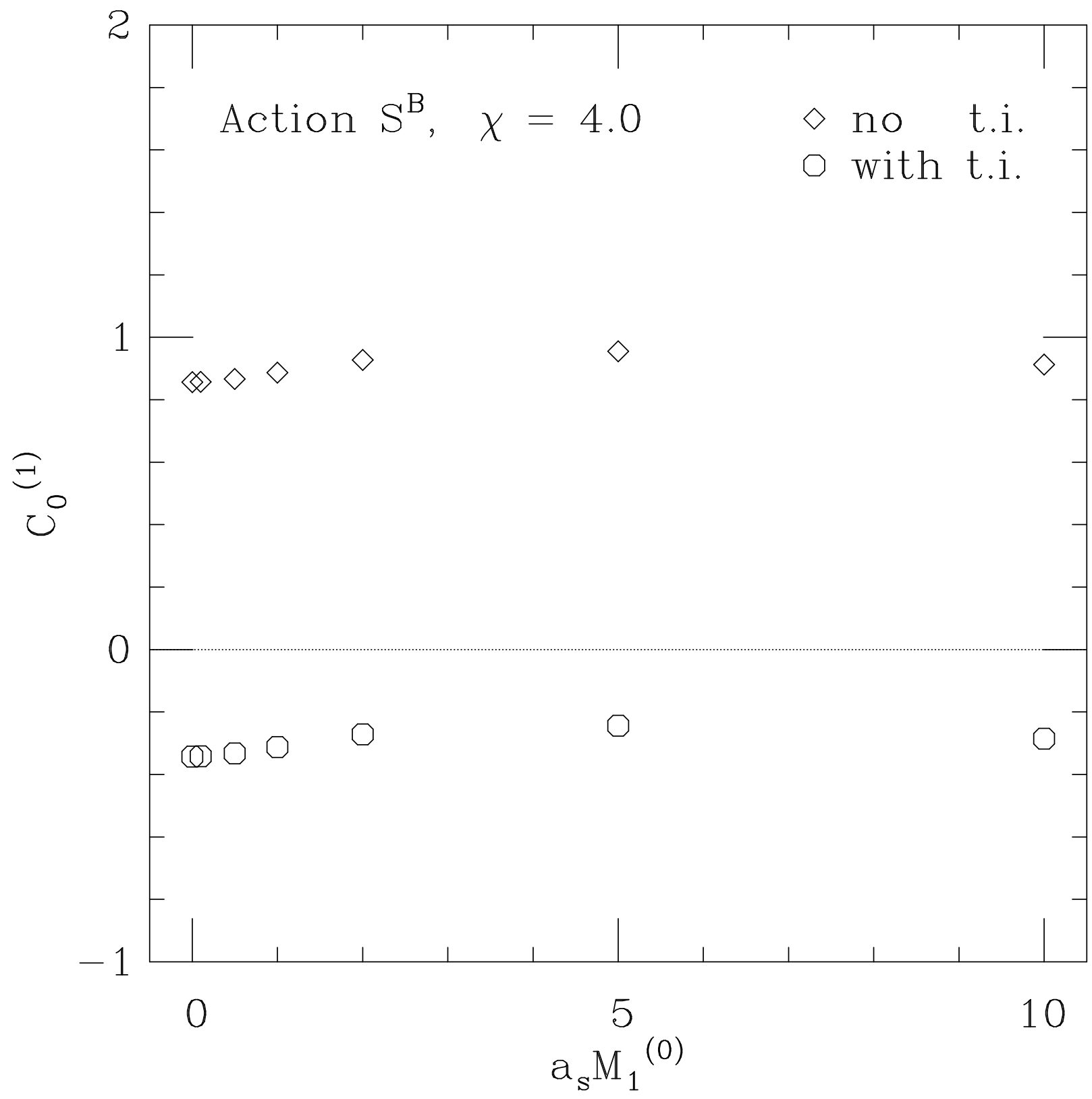

FIG. 3. $C_{0}^{(1)}$ versus $a_{s} M_{1}^{(0)}$ both with and without tadpole improvement for action $\mathcal{S}^{B}$. 


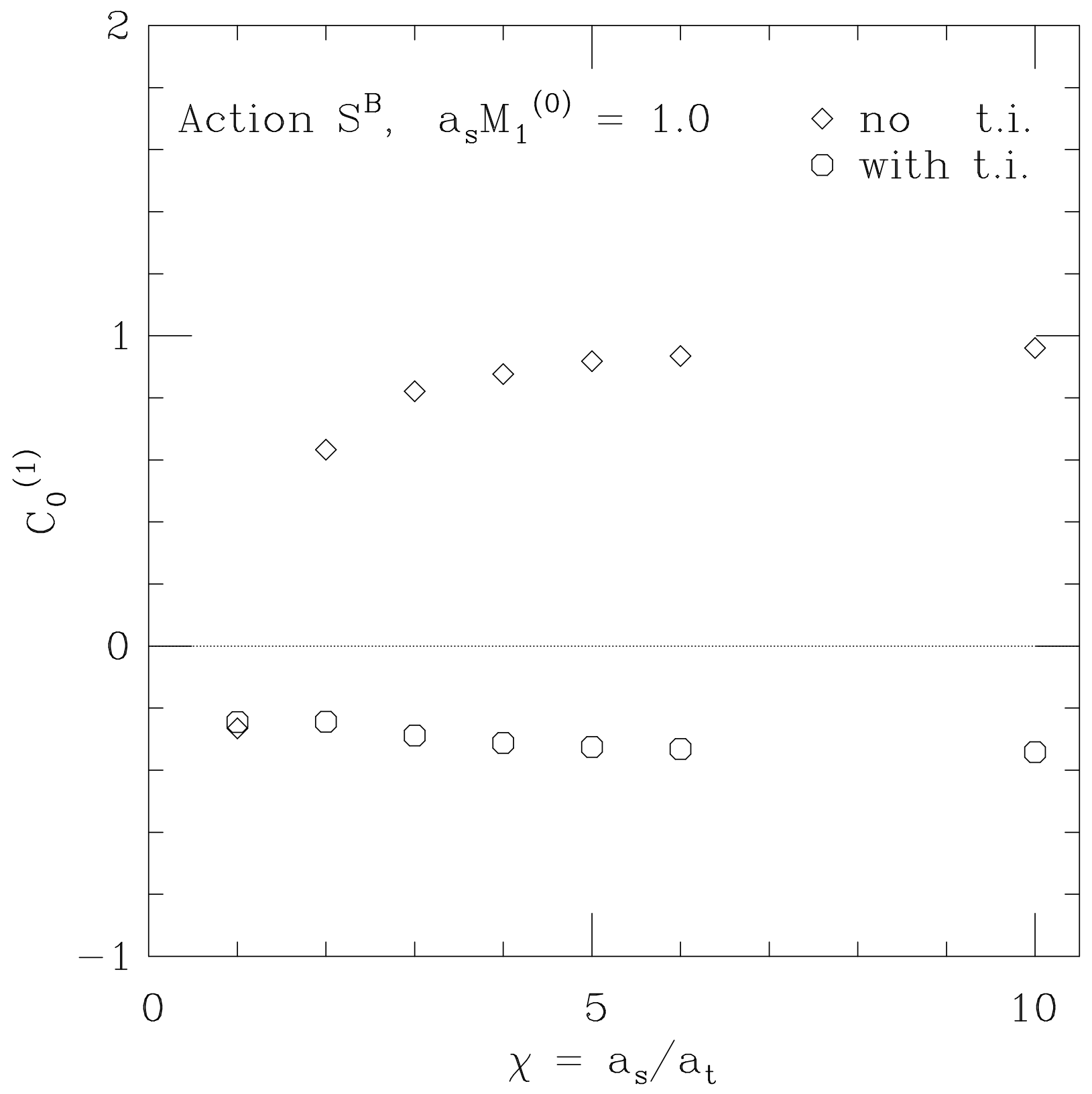

FIG. 4. $C_{0}^{(1)}$ versus $\chi$ both with and without tadpole improvement for action $\mathcal{S}^{B}$. 